\title{
Nonlocal Lazer-McKenna-type problem perturbed by the Hardy's potential and its parabolic equivalence
}

\author{
Masoud Bayrami-Aminlouee ${ }^{1 *}$ (D, Mahmoud Hesaaraki ${ }^{1}$, Mohamed Karim Hamdani ${ }^{2,3,4}$ and
}

Nguyen Thanh Chung ${ }^{5}$

"Correspondence:

masoud.bayrami1990@student.sharif.edu

'Department of Mathematical

Sciences, Sharif University of

Technology, Azadi Ave, P.O. Box

11365-9415, Tehran, Iran

Full list of author information is available at the end of the article

\section{Abstract}

In this paper, we study the effect of Hardy potential on the existence or nonexistence of solutions to the following fractional problem involving a singular nonlinearity:

$$
\begin{cases}(-\Delta)^{s} u=\lambda \frac{u}{|x|^{2 s}}+\frac{\mu}{u \gamma}+f & \text { in } \Omega, \\ u>0 & \text { in } \Omega, \\ u=0 & \text { in }\left(\mathbb{R}^{N} \backslash \Omega\right) .\end{cases}
$$

Here $0<s<1, \lambda>0, \gamma>0$, and $\Omega \subset \mathbb{R}^{N}(N>2 s)$ is a bounded smooth domain such that $0 \in \Omega$. Moreover, $0 \leq \mu, f \in L^{1}(\Omega)$. For $0<\lambda \leq \Lambda_{N, S}, \Lambda_{N, s}$ being the best constant in the fractional Hardy inequality, we find a necessary and sufficient condition for the existence of a positive weak solution to the problem with respect to the data $\mu$ and $f$. Also, for a regular datum of $f$, under suitable assumptions, we obtain some existence and uniqueness results and calculate the rate of growth of solutions. Moreover, we mention a nonexistence and a complete blowup result for the case $\lambda>\Lambda_{N, s}$. Besides, we consider the parabolic equivalence of the above problem in the case $\mu \equiv 1$ and some suitable $f(x, t)$, that is,

$$
\begin{cases}u_{t}+(-\Delta)^{s} u=\lambda \frac{u}{|x|^{2 s}}+\frac{1}{u^{\gamma}}+f(x, t) & \text { in } \Omega \times(0, T) \\ u>0 & \text { in } \Omega \times(0, T), \\ u=0 & \text { in }\left(\mathbb{R}^{N} \backslash \Omega\right) \times(0, T), \\ u(x, 0)=u_{0} & \text { in } \mathbb{R}^{N},\end{cases}
$$

where $u_{0} \in X_{0}^{s}(\Omega)$ satisfies an appropriate cone condition. In the case $0<\gamma \leq 1$ or $\gamma>1$ with $2 s(\gamma-1)<(\gamma+1)$, we show the existence of a unique solution for any $0<\lambda<\Lambda_{N, s}$ and prove a stabilization result for certain range of $\lambda$.

MSC: 35R11;35B25; 35A01; 35B09; 35B44

Keywords: Singular fractional Laplacian heat equation; Hardy singularity; Singular nonlinearity; Existence and nonexistence; Positive solution; Blowup

\section{Springer}

(c) The Author(s) 2021. This article is licensed under a Creative Commons Attribution 4.0 International License, which permits use, sharing, adaptation, distribution and reproduction in any medium or format, as long as you give appropriate credit to the original author(s) and the source, provide a link to the Creative Commons licence, and indicate if changes were made. The images or other third party material in this article are included in the article's Creative Commons licence, unless indicated otherwise in a credit line to the material. If material is not included in the article's Creative Commons licence and your intended use is not permitted by statutory regulation or exceeds the permitted use, you will need to obtain permission directly from the copyright holder. To view a copy of this licence, visit http://creativecommons.org/licenses/by/4.0/. 


\section{Introduction}

We study the existence and nonexistence of positive solutions to the following singular elliptic problem:

$$
\begin{cases}(-\Delta)^{s} u=\lambda \frac{u}{|x|^{2 s}}+\frac{\mu}{u^{\gamma}}+f & \text { in } \Omega, \\ u>0 & \text { in } \Omega, \\ u=0 & \text { in }\left(\mathbb{R}^{N} \backslash \Omega\right) .\end{cases}
$$

Here $\Omega \subset \mathbb{R}^{N}, N>2 s$, is an open bounded domain with smooth boundary such that $0 \in \Omega$, $s \in(0,1), \lambda>0$, and $\gamma>0$. Moreover, $0 \leq \mu, f \in L^{1}(\Omega)$.

We will prove that for $0<\lambda \leq \Lambda_{N, s}, \Lambda_{N, s}=\frac{4^{s} \Gamma^{2}\left(\frac{N+2 s}{4}\right)}{\Gamma^{2}\left(\frac{N-2 s}{4}\right)}$ being the best constant in the fractional Hardy inequality, the problem has a solution if and only if $\mu \in L^{1}\left(\Omega, \delta^{s(1-\gamma)} d x\right)$, $\delta(x)=\operatorname{dist}(x, \partial \Omega)$, and the datum of $f$ satisfies the following integrability condition:

$$
\int_{\Omega} f(x)|x|^{-\beta} d x<+\infty
$$

where the constant $\beta=\beta(N, s, \lambda)$ will be defined later in Lemma 2.4. In this lemma, we will see that any supersolution to (1) is unbounded near the origin and the nature of this unboundedness is like $u(x) \gtrsim|x|^{-\beta}$ in some open ball centered at the origin.

Also, we will see that there is no positive very weak (distributional) solution for the case $\lambda>\Lambda_{N, s}$. This notion of the solution, which we consider for the nonexistence result, is local in nature, and we just ask the regularity needed to give distributional sense to the equation (similar to that in $[1,2])$. Moreover, this nonexistence result is strong in the sense that a complete blowup phenomenon occurs. By complete blowup phenomenon we mean that the solutions to the approximating problems (with the bounded weights $\left(|x|^{2 s}+\epsilon\right)^{-1}$ and $(u+\epsilon)^{-\gamma}$ instead of the terms $|x|^{-2 s}$ and $u^{-\gamma}$, respectively) tend to infinity for every $x \in \Omega$ as $0<\epsilon \downarrow 0$.

In the above problem, $(-\Delta)^{s}$ stands for the fractional Laplacian operator:

$$
\begin{aligned}
(-\Delta)^{s} u(x) & =C_{N, s} \text { P.V. } \int_{\mathbb{R}^{N}} \frac{u(x)-u(y)}{|x-y|^{N+2 s}} d y \\
& =C_{N, s} \lim _{\epsilon \rightarrow 0^{+}} \int_{|x-y| \geq \epsilon} \frac{u(x)-u(y)}{|x-y|^{N+2 s}} d y, \quad u \in \mathcal{S}\left(\mathbb{R}^{N}\right),
\end{aligned}
$$

where P.V. is a commonly used abbreviation for the Cauchy principal value and is defined by the latter equation. Also, $\mathcal{S}\left(\mathbb{R}^{N}\right)$ denotes the Schwartz space (space of "rapidly decreasing functions" on $\left.\mathbb{R}^{N}\right)$, and $C_{N, s}=\frac{4^{s} \Gamma\left(\frac{N}{2}+s\right)}{\pi^{\frac{N}{2}}|\Gamma(-s)|}$ is the normalization constant such that

$$
(-\Delta)^{s} u=\mathcal{F}^{-1}\left(|\xi|^{2 s} \hat{u}(\xi)\right)
$$

Here $\Gamma$ denotes the gamma function, and $\mathcal{F} u=\hat{u}$ is the Fourier transform of $u$. By restricting the fractional Laplacian operator to act only on smooth functions that are zero outside $\Omega$, we have the restricted fractional Laplacian $\left(-\Delta_{I_{\Omega}}\right)^{s}$. For this operator, the best alternative to the Dirichlet boundary condition is $u \equiv 0$ in $\left(\mathbb{R}^{N} \backslash \Omega\right)$. For more details about fractional Laplacian, see [3-5]. 
Over the past decades, there has been much focus and also a vast literature on singular problems. Singularities appear in almost all fields of mathematics like differential geometry and partial differential equations. Singularities are the qualitative side of mathematics, and understanding of singularities always leads to a more detailed picture of the objects mathematics is dealing with [6]. Many more details and references for the singular elliptic problems can be found in [7].

One famous type of singularities is the singularity of Hardy type, which is related to the inequality of the same name, and there are various its generalizations. The well-known classical Hardy inequality is as follows:

$$
\int_{\Omega}|\nabla u|^{p} d x \geq\left(\frac{N-p}{p}\right)^{p} \int_{\Omega} \frac{|u|^{p}}{|x|^{p}} d x, \quad u \in W_{0}^{1, p}(\Omega)
$$

where $\Omega \subset \mathbb{R}^{N}$ is a bounded domain containing the origin, and $1 \leq p<N[8,9]$. The constant $\left(\frac{N-p}{p}\right)^{p}$ is optimal, and it is not attained in $W_{0}^{1, p}(\Omega)$, meaning that the continuous embedding $W_{0}^{1, p}(\Omega) \hookrightarrow L^{p}\left(\Omega,|x|^{-p} d x\right)$ is not compact. The intention of analyzing Hardy singularities has come from its widespread use in different branches of science. For details and references about the enormous literature for this topic, see the more recent book [10] and Chap. 1 of [11]. Due to these motivations, over the past few decades, general singularities are widely studied.

In the pioneering works, Baras and Goldstein $[12,13]$ studied the following singular Cauchy-Dirichlet heat problem in $\Omega=\mathbb{R}^{N}$ or in a bounded smooth domain $\Omega$ containing $B_{1}(0)=\left\{x \in \mathbb{R}^{N}:\|x\|<1\right\}:$

$$
\begin{cases}\frac{\partial u}{\partial t}-\Delta u=V(x) u+f(x, t), & (x, t) \in \Omega \times(0, \infty), \\ u(x, t)=0, & (x, t) \in \partial \Omega \times(0, \infty), \\ u(x, 0)=u_{0}(x), & x \in \Omega .\end{cases}
$$

The authors assume that $f$ and $u_{0}$ are nonnegative and $0 \leq V \in L^{\infty}\left(\Omega \backslash B_{\epsilon}(0)\right)$ for each $\epsilon>0$, but $V$ may be singular at the origin. They say that $V$ is too singular if $V(x)>\frac{C^{*}(N)}{|x|^{2}}$ near $x=0$, whereas $V$ is not too singular if $V(x) \leq \frac{C^{*}(N)}{|x|^{2}}$ near $x=0$. Here $C^{*}(N)=\frac{(N-2)^{2}}{4}$ is the sharp constant in the following Hardy inequality:

$$
C^{*}(N) \int_{\Omega} \frac{u^{2}}{|x|^{2}} d x \leq \int_{\Omega}|\nabla u|^{2} d x, \quad \forall u \in H_{0}^{1}(\Omega)
$$

In the not too singular potential case, they found necessary and sufficient conditions for the existence of a nonnegative distributional solution to problem (2). Moreover, they obtained this solution as the limit of the solutions to the following approximate problem:

$$
\begin{cases}\frac{\partial u_{n}}{\partial t}-\Delta u_{n}=V_{n}(x) u_{n}+f(x, t), & (x, t) \in \Omega \times(0, \infty), \\ u_{n}(x, t)=0, & (x, t) \in \partial \Omega \times(0, \infty), \\ u_{n}(x, 0)=u_{0}(x), & x \in \Omega,\end{cases}
$$

where $V_{n}(x)=\min \{V(x), n\}$. Also, for the too singular potential case, they showed that the problem has no solution even in the sense of distributions and an instantaneous complete blowup phenomenon occurs. Namely, $u_{n}(x, t) \rightarrow+\infty$ for all $(x, t) \in \Omega \times(0, T)$ as $n \rightarrow \infty$. 
In problem (1) the singular term $\frac{\lambda}{|x|^{2 s}}$ is related to the following fractional Hardy inequality:

$$
\Lambda_{N, s} \int_{\mathbb{R}^{N}} \frac{|u(x)|^{2}}{|x|^{2 s}} d x \leq \int_{\mathbb{R}^{N}}\left|(-\Delta)^{\frac{s}{2}} u(x)\right|^{2} d x, \quad \forall u \in C_{c}^{\infty}\left(\mathbb{R}^{N}\right)
$$

where $N>2 s, s \in(0,1)$, and the constant $\Lambda_{N, s}=\frac{4^{s} \Gamma^{2}\left(\frac{N+2 s}{4}\right)}{\Gamma^{2}\left(\frac{N-2 s}{4}\right)}$ is optimal [14]. Problem (1) is motivated by the papers $[15,16]$, in which the authors proved the existence of solutions to the following Lazer-McKenna-type problem:

$$
\begin{cases}-\Delta u=\frac{\mu}{u^{\gamma}} & \text { in } \Omega, \\ u>0 & \text { in } \Omega, \\ u=0 & \text { on } \partial \Omega,\end{cases}
$$

where $\Omega$ is a bounded domain of $\mathbb{R}^{N}, N \geq 2, \gamma>0$, and $\mu$ is a general Radon measure in $\Omega$. See [17-26] for more related problems. These types of problems have been extensively studied for their relations with some physical phenomena in the theory of pseudoplastic fluids [27].

Barrios, Bonis, Medina, and Peral [17] studied the solvability of the following superlinear problem:

$$
\begin{cases}(-\Delta)^{s} u=\lambda \frac{f(x)}{u^{\gamma}}+M u^{p} & \text { in } \Omega, \\ u>0 & \text { in } \Omega, \\ u=0 & \text { in }\left(\mathbb{R}^{N} \backslash \Omega\right) .\end{cases}
$$

More precisely, for the case $M=0$ and $f \geq 0$, they proved the existence of a positive solution for all $\gamma>0$ and $\lambda>0$. Moreover, in the case $M=1$ and $f \equiv 1$, they found a threshold $\Lambda$ such that there exists a solution for every $0<\lambda<\Lambda$ and there does not for $\lambda>\Lambda$. Also, in [28] the authors considered the similar superlinear problem with the critical growth, namely when $p=2_{s}^{*}-1=\frac{N+2 s}{N-2 s}$, and with a singular nonlinearity of the form $u^{-q}, q \in(0,1)$. In the detailed paper [29], Abdellaoui, Medina, Peral, and Primo studied the effect of the Hardy potential on the existence and summability of the solutions to a class of fractional Laplacian problems. We will use the essential tool introduced in this paper, that is, the weak Harnack inequality, which they proved by following the classical Moser and Krylov-Safonov idea. Also, we will take advantage of some of Calderón-Zygmund properties of solutions. See [29, Sect. 4] for the effect of the Hardy potential in some CalderónZygmund properties for the fractional Laplacian.

For the similar parabolic equivalence of (1), Giacomoni, Mukherjee, and Sreenadh [30] investigated the existence and stabilization for the following parabolic equation involving the fractional Laplacian with singular nonlinearity:

$$
\begin{cases}u_{t}+(-\Delta)^{s} u=u^{-q}+f(x, u) & \text { in } \Omega \times(0, T), \\ u(x, 0)=u_{0}(x) & \text { in } \mathbb{R}^{N}, \\ u(x, t)>0 & \text { in } \Omega \times(0, T), \\ u(x, t)=0 & \text { in } \left.\mathbb{R}^{N} \backslash \Omega\right) \times(0, T) .\end{cases}
$$


Under suitable assumptions on the parameters and datum, they studied the related stationary problem, and then using a semidiscretization in time with the implicit Euler method, they proved the existence and uniqueness of the weak solution. It is worth noting that in $[31,32]$ authors have shown the same results for the local version of this problem for the general $p$-Laplacian case. Also, for some recent papers on the optimal regularity, see $[33,34]$.

The rest of the paper is as follows. In Sect. 2, after introducing the functional setting, we outline our existence and nonexistence theorems. In particular, we provide a theorem about necessary and sufficient conditions for the existence of a solution to problem (1) in the case $\lambda \leq \Lambda_{N, s}$ and a nonexistence theorem in the case $\lambda>\Lambda_{N, s}$. In Sect. 3, we provide proofs of our existence theorems. In Sect. 4, we will present some uniqueness results. Also, concerning uniqueness, with some regularity assumptions on $\mu$ and $f$, we show the existence and uniqueness of the so-called entropy solution for the case $0<\gamma \leq 1$. Besides, we mention a theorem about the rate of growth of solutions to problem (1). Finally, in Sect. 5, we consider the parabolic version of problem (1) in the particular case $\mu \equiv 1$. Firstly, under the assumptions $0<\gamma \leq 1$ or $\gamma>1$ with $2 s(\gamma-1)<(\gamma+1)$, we show the existence of a unique solution for $0<\lambda<\Lambda_{N, s}$ and secondly, we prove the stability for some range of $\lambda$; that is, we find a positive constant $\lambda_{*}=\lambda_{*}(N, s)<\Lambda_{N, s}$ such that for any $\lambda \in\left(0, \lambda_{*}\right)$, the solution to the parabolic problem converges to the unique solution of its stationary problem as $t \rightarrow \infty$.

\section{Functional setting and existence, nonexistence, and blowup results}

Let $0<s<1,1 \leq p<\infty$, and let $\Omega$ be a bounded domain in $\mathbb{R}^{N}$. Also, let $D_{\Omega}=\mathbb{R}^{N} \times \mathbb{R}^{N} \backslash$ $\Omega^{c} \times \Omega^{c}$ with $\Omega^{c}=\mathbb{R}^{N} \backslash \Omega$. We define the Banach space

$$
X^{s, p}(\Omega)=\left\{u: \mathbb{R}^{N} \rightarrow \mathbb{R} \text { measurable, }\left.u\right|_{\Omega} \in L^{p}(\Omega), \iint_{D_{\Omega}} \frac{|u(x)-u(y)|^{p}}{|x-y|^{N+p s}} d x d y<\infty\right\}
$$

endowed with the norm

$$
\|u\|_{X^{s, p}(\Omega)}=\left(\int_{\Omega}|u|^{p} d x+\iint_{D_{\Omega}} \frac{|u(x)-u(y)|^{p}}{|x-y|^{N+p s}} d x d y\right)^{\frac{1}{p}} .
$$

In the case $p=2$, we denote by $X^{s}(\Omega)$ the space $X^{s, 2}(\Omega)$, which is a Hilbert space with the inner product

$$
\langle u, v\rangle_{X^{s}(\Omega)}=\int_{\Omega} u v d x+\iint_{D_{\Omega}} \frac{(u(x)-u(y))(v(x)-v(y))}{|x-y|^{N+2 s}} d x d y .
$$

Moreover, we define $X_{0}^{s, p}(\Omega)$ as the closure of $C_{0}^{\infty}(\Omega)$ in $X^{s, p}\left(\mathbb{R}^{N}\right)$. Equivalently, it can be shown that

$$
X_{0}^{s, p}(\Omega)=\left\{u \in X^{s, p}\left(\mathbb{R}^{N}\right): u=0 \text { a.e. in }\left(\mathbb{R}^{N} \backslash \Omega\right)\right\} .
$$

It is easy to see that

$$
\left(\int_{\mathbb{R}^{N}} \int_{\mathbb{R}^{N}} \frac{|u(x)-u(y)|^{p}}{|x-y|^{N+p s}} d x d y\right)^{\frac{1}{p}}=\left(\iint_{D_{\Omega}} \frac{|u(x)-u(y)|^{p}}{|x-y|^{N+p s}} d x d y\right)^{\frac{1}{p}}, \quad \forall u \in X_{0}^{s}(\Omega) .
$$


This equality defines an equivalent norm for $X_{0}^{s, p}(\Omega)$ with (4). We denote it by

$$
\|u\|_{X_{0}^{s, p}(\Omega)}=\left(\iint_{D_{\Omega}} \frac{|u(x)-u(y)|^{p}}{|x-y|^{N+p s}} d x d y\right)^{\frac{1}{p}}
$$

It is worth noting that the continuous embedding of $X_{0}^{s_{2}}(\Omega)$ into $X_{0}^{s_{1}}(\Omega)$ holds for any $s_{1}<s_{2}$ (see, e.g., [4, Proposition 2.1]). Besides, for the Hilbert space case, we have

$$
\|u\|_{X_{0}^{s}(\Omega)}^{2}=2 C_{N, s}^{-1}\left\|(-\Delta)^{\frac{s}{2}} u\right\|_{L^{2}\left(\mathbb{R}^{N}\right)}^{2}
$$

where $C_{N, s}$ is the normalization constant in the definition of $(-\Delta)^{s}$. Thus Hardy inequality (3) also can be written as follows:

$$
\Lambda_{N, s} \int_{\mathbb{R}^{N}} \frac{|u(x)|^{2}}{|x|^{2 s}} d x \leq \frac{C_{N, s}}{2} \iint_{D_{\Omega}} \frac{|u(x)-u(y)|^{2}}{|x-y|^{N+2 s}} d x d y, \quad \forall u \in X_{0}^{s}(\Omega)
$$

For the proofs of the above facts, see [35, Sect. 2.2] and [4]. Also, see [36, Sect. 2].

In this paper, we will use the following continuous embedding:

$$
X_{0}^{s, p}(\Omega) \hookrightarrow L^{q}(\Omega), \quad \forall q \in\left[1, p_{s}^{*}\right]
$$

where $p_{s}^{*}=\frac{p N}{N-p s}$ is the Sobolev critical exponent. Moreover, this embedding is compact for $1 \leq q<p_{s}^{*}$. See [4, Theorems 6.5 and 7.1].

Also, we denote by $X_{\text {loc }}^{s, p}(\Omega)$ the set of all functions $u$ such that $u \phi \in X_{0}^{s, p}(\Omega)$ for any $\phi \in$ $C_{c}^{\infty}(\Omega)$. When we say that $\left\{u_{n}\right\} \subset X_{\text {loc }}^{s, p}(\Omega)$ is bounded, we mean that $\left\{\phi u_{n}\right\} \subset X_{0}^{s, p}(\Omega)$ is bounded for any fixed $\phi \in C_{c}^{\infty}(\Omega)$.

Since we are dealing with the nonlocal operator $(-\Delta)^{s}$, we will use the following class of test functions for defining the weak solution to problem (1):

$$
\mathcal{T}(\Omega)=\left\{\begin{array}{l|l}
\phi: \mathbb{R}^{N} \rightarrow \mathbb{R} \mid \begin{array}{l}
(-\Delta)^{s} \phi=\varphi, \varphi \in L^{\infty}(\Omega) \cap C^{0, \alpha}(\Omega), 0<\alpha<1, \\
\phi=0 \text { in }\left(\mathbb{R}^{N} \backslash \Omega\right)
\end{array}
\end{array}\right\}
$$

It can be shown that $\mathcal{T}(\Omega) \subset X_{0}^{s}(\Omega) \cap L^{\infty}(\Omega) \cap C^{0, s}(\Omega)$. See [29], where this class of test functions is used for dealing with problem (1). Moreover, every $\phi \in \mathcal{T}(\Omega)$ is a strong solution to the equation $(-\Delta)^{s} \phi=\varphi$, and for every $\phi \in \mathcal{T}(\Omega)$, there exists a constant $\beta \in(0,1)$ such that $\frac{\phi}{\delta^{s}} \in C^{0, \beta}(\Omega)$; see [37].

It is easy to check that for $u \in X_{0}^{s}(\Omega)$ and $\phi \in \mathcal{T}(\Omega)$,

$$
\begin{aligned}
2 C_{N, s}^{-1} \int_{\mathbb{R}^{N}} u(-\Delta)^{s} \phi d x & =2 C_{N, s}^{-1} \int_{\mathbb{R}^{N}}(-\Delta)^{\frac{s}{2}} u(-\Delta)^{\frac{s}{2}} \phi d x \\
& =\iint_{D_{\Omega}} \frac{(u(x)-u(y))(\phi(x)-\phi(y))}{|x-y|^{N+2 s}} d x d y .
\end{aligned}
$$

One can show that $(-\Delta)^{s}: X_{0}^{s}(\Omega) \rightarrow X^{-s}(\Omega)$ is a continuous strictly monotone operator, where $X^{-s}(\Omega)$ is the dual space of $X_{0}^{s}(\Omega)$. 
Definition 2.1 We say that $u \in L^{1}(\Omega)$ is a very weak (distributional) supersolution (subsolution) to

$$
(-\Delta)^{s} u=g(x, u) \text { in } \Omega
$$

if $g(x, u) \in L^{1}(\Omega), u \equiv 0$ in $\left(\mathbb{R}^{N} \backslash \Omega\right)$, and $(-\Delta)^{s} u \geq(\leq) g(x, u)$ in the weak sense, that is,

$$
\int_{\mathbb{R}^{N}} u(-\Delta)^{s} \phi d x \geq(\leq) \int_{\Omega} g(x, u) \phi d x
$$

for all nonnegative $\phi \in \mathcal{T}(\Omega)$. If $u$ is a very weak (distributional) supersolution and subsolution, then we say that $u$ is a very weak (distributional) solution.

Definition 2.2 We say that $u \in X_{0}^{s}(\Omega)$ is a weak energy supersolution (subsolution) to

$$
(-\Delta)^{s} u=g(x, u) \text { in } \Omega
$$

if $g(x, u) \in X_{0}^{s}(\Omega), u \equiv 0$ in $\left(\mathbb{R}^{N} \backslash \Omega\right)$, and $(-\Delta)^{s} u \geq(\leq) g(x, u)$ in the weak sense, that is,

$$
\int_{\mathbb{R}^{N}} u(-\Delta)^{s} \phi d x \geq(\leq) \int_{\Omega} g(x, u) \phi d x
$$

for all nonnegative $\phi \in X_{0}^{s}(\Omega)$. If $u$ is a weak energy supersolution and subsolution, then we say that $u$ is a weak energy solution.

Definition 2.3 Let $0 \leq \mu, f \in L^{1}(\Omega)$. We say that $u$ is a weak solution to problem (1) if

- $u \in L^{1}(\Omega)$, and for every $K \Subset \Omega$, there exists $C_{K}>0$ such that $u(x) \geq C_{K}$ a.e. in $K$ and also $u \equiv 0$ in $\left(\mathbb{R}^{N} \backslash \Omega\right)$;

- Equation (1) is satisfied in the weak sense, that is,

$$
\int_{\mathbb{R}^{N}} u(-\Delta)^{s} \phi d x=\lambda \int_{\Omega} \frac{u \phi}{|x|^{2 s}} d x+\int_{\Omega} \frac{\mu \phi}{u^{\gamma}} d x+\int_{\Omega} f \phi d x, \quad \forall \phi \in \mathcal{T}(\Omega),
$$

together with extra assumption that the first and second terms on the right-hand side of this equality are finite for any $\phi \in \mathcal{T}(\Omega)$. The well-posedness of the first and second terms on the right-hand side will be clear after the construction of solution.

Remark 1 Plugging the test function $\phi=\psi_{1, s}$ into (8), where $\psi_{1, s}$ is the normalized first eigenfunction associated with first eigenvalue $\lambda_{1, s}$ of $(-\Delta)^{s}$ in $X_{0}^{s}(\Omega)$, that is,

$$
\begin{cases}(-\Delta)^{s} \psi_{1, s}=\lambda_{1, s} \psi_{1, s} & \text { in } \Omega, \\ \psi_{1, s}=0 & \text { in }\left(\mathbb{R}^{N} \backslash \Omega\right), \\ 0<\psi_{1, s} \in X_{0}^{s}(\Omega) \cap L^{\infty}(\Omega), & \\ \left\|\psi_{1, s}\right\|_{L^{2}(\Omega)}=1 & \end{cases}
$$

(see, e.g., [38, Proposition 9]) and also noting that there exist $l_{1}, l_{2}>0$ such that $l_{1} \delta^{s}(x) \leq$ $\psi_{1, s}(x) \leq l_{2} \delta^{s}(x)$ for a.e. $x \in \Omega$ [37], we obtain that the solution $u$ necessarily satisfies

$$
\int_{\Omega} \frac{\mu}{u^{\nu}} \delta^{s} d x<+\infty
$$


Moreover, since by the comparison principle for the fractional Laplacian and Hopf's lemma, $u \geq c \delta^{s}$ a.e. in $\Omega$ (see, e.g., [39] or [40, Lemma 4.2]), we have

$$
\int_{\Omega} \frac{\mu}{\delta^{s(\gamma-1)}} d x<+\infty
$$

As an analysis of the linear case with Hardy potential, we first gather the following lemmas.

Lemma 2.4 Let $\lambda \leq \Lambda_{N, s}$. Let $u$ be a nonnegative function defined in $\Omega$ such that $u \neq \equiv$, $u \in L^{1}(\Omega), \frac{u}{|x|^{2 s}} \in L^{1}(\Omega)$, and $u \geq 0$ in $\left(\mathbb{R}^{N} \backslash \Omega\right)$. If $u$ satisfies $(-\Delta)^{s} u-\lambda \frac{u}{|x|^{2 s}} \geq 0$ in the weak sense in $\Omega$, then there exist $\delta>0$ and a constant $C=C(N, \delta)$ such that

$$
u \geq C|x|^{-\beta} \quad \text { in } B_{\delta}(0)
$$

where $\beta=\frac{N-2 s}{2}-\alpha$, and $\alpha$ is given by the identity

$$
\lambda=\frac{4^{s} \Gamma\left(\frac{N+2 s+2 \alpha}{4}\right) \Gamma\left(\frac{N+2 s-2 \alpha}{4}\right)}{\Gamma\left(\frac{N-2 s+2 \alpha}{4}\right) \Gamma\left(\frac{N-2 s-2 \alpha}{4}\right)} .
$$

Lemma 2.5 Let $\lambda \leq \Lambda_{N, s}$. Let $u$ be a positive very weak solution to

$$
\begin{cases}(-\Delta)^{s} u-\lambda \frac{u}{|x|^{2 s}}=g & \text { in } \Omega, \\ u>0 & \text { in } \Omega, \\ u=0 & \text { in }\left(\mathbb{R}^{N} \backslash \Omega\right),\end{cases}
$$

with $g \in L^{1}(\Omega)$ and $g \geq 0$. Then $g|x|^{-\beta} \in L^{1}\left(B_{r}(0)\right)$ for some $B_{r}(0) \Subset \Omega$.

Lemma 2.6 (Weak Harnack inequality) Let $r>0$ be such that $B_{2 r}(0) \subset \Omega$. Assume that $f \geq 0$, and let $v \in X_{0}^{s}(\Omega)$ with $v \supsetneqq 0$ in $\mathbb{R}^{N}$ be a supersolution to

$$
\begin{cases}(-\Delta)^{s} v=f & \text { in } \Omega, \\ v=0 & \text { in }\left(\mathbb{R}^{N} \backslash \Omega\right),\end{cases}
$$

that is,

$$
\int_{\mathbb{R}^{N}}(-\Delta)^{\frac{s}{2}} v(-\Delta)^{\frac{s}{2}} \phi d x \geq \int_{\Omega} f \phi d x
$$

for all nonnegative $\phi \in X_{0}^{s}(\Omega)$. Then, for every $q<\frac{N}{N-2 s}$, there exists a positive constant $C=C(N, s)$ such that

$$
\left(\int_{B_{r}(0)} v^{q} d x\right)^{\frac{1}{q}} \leq C \inf _{B_{\frac{3}{2}}(0)} v .
$$

For the proof of these lemmas, see [29, Lemma 3.10], [29, Theorem 4.10], and [29, Theorem 3.4], respectively. 
In the next two theorems, we present our existence results to problem (1). First, we will prove that for $0<\lambda<\Lambda_{N, s}$ and $\gamma \geq 1$, problem (1) admits a solution for the case $\mu \epsilon$ $L^{1}(\Omega)$ and $f \in L^{1}(\Omega) \cap X^{-s}(\Omega)$. It is crucial to indicate that our approach in the proof of Theorem 2.7 only works in the case $\gamma \geq 1$. However, if we further assume that $\mu \in L^{m}(\Omega)$, $m=\left(\frac{2_{s}^{*}}{1-\gamma}\right)^{\prime}\left(p^{\prime}\right.$ denotes the conjugate exponent of $\left.p\right)$, then the same approach works for $\gamma<$ 1. For a result on the existence with less regularity assumption on $\mu$, see [29, Theorem 5.3]. More precisely, the authors showed an existence result for the case $\mu \in L^{1}\left(\Omega,|x|^{-(1-\gamma) \beta} d x\right)$.

In the following, we denote

$$
T_{n}(\sigma)= \begin{cases}\sigma, & |\sigma| \leq n \\ n \frac{\sigma}{|\sigma|}, & |\sigma| \geq n\end{cases}
$$

the usual truncation operator, and $G_{n}(\sigma):=\sigma-T_{n}(\sigma)$.

Theorem 2.7 Let $s \in(0,1), 0<\lambda<\Lambda_{N, s}=\frac{4^{s} \Gamma^{2}\left(\frac{N+2 s}{4}\right)}{\Gamma^{2}\left(\frac{N-2 s}{4}\right)}$, and $\gamma>0$. Also, let $\mu \in L^{1}(\Omega)$ be a nonnegative function, and let $0 \leq f \in L^{1}(\Omega) \cap X^{-s}(\Omega)$.

1. If $\gamma=1$, then there is a positive weak solution in $X_{0}^{s}(\Omega)$ to problem (1).

2. If $\gamma>1$, then there is a positive weak solution in $X_{\mathrm{loc}}^{s}(\Omega)$ to problem (1) with $T_{k}^{\frac{\gamma+1}{2}}(u) \in X_{0}^{s}(\Omega)$ and $G_{k}(u) \in X_{0}^{s}(\Omega)$. In addition, if $\frac{4 \gamma}{(\gamma+1)^{2}}>\frac{\lambda}{\Lambda_{N, s}}$, then $u^{\frac{\gamma+1}{2}} \in X_{0}^{s}(\Omega)$.

3. If $\gamma<1$ and $\mu \in L^{\left(\frac{2 *}{1-\gamma}\right)^{\prime}}(\Omega)$, then there is a positive weak solution in $X_{0}^{s}(\Omega)$ to problem (1).

The next theorem gives a necessary and sufficient condition for the existence of a solution to problem (1).

Theorem 2.8 (A necessary and sufficient condition for the existence of a solution) Let $s \in(0,1), 0<\lambda \leq \Lambda_{N, s}$, and $\gamma>0$. Also, let $0 \leq f, \mu \in L^{1}(\Omega)$. Then problem (1) has a positive weak solution if and only if

$$
\int_{\Omega} \frac{f(x)}{|x|^{\beta}} d x<+\infty, \quad \int_{\Omega} \frac{\mu}{\delta^{s(\gamma-1)}} d x<+\infty .
$$

Moreover, the solution $u$ has the following regularity:

- $T_{k}(u) \in X_{0}^{s}(\Omega)$ for all $k>0$, and $u \in L^{p}(\Omega)$ for all $p \in\left[1, \frac{N}{N-2 s}\right)$.

- $(-\Delta)^{\frac{s}{2}} u \in L^{p}(\Omega)$ for all $p \in\left[1, \frac{N}{N-s}\right)$.

- $u \in X_{0}^{s_{1}, p}(\Omega)$ for all $s_{1}<s$ and $p<\frac{N}{N-s}$.

Remark 2 A similar argument as in [41, Example 3.3] but with the fractional Laplacian instead of the Laplacian operator shows that problem (1) does not admit a solution for merely $f \in L^{1}(\Omega)$.

The proof of these theorems will appear in the next section. In the following, we will have a nonexistence and also a blowup result for the case $\lambda>\Lambda_{N, s}$.

The following nonexistence result is an immediate consequence of Lemmas 2.4 and 2.5. More precisely, it is well known that the linear problem with Hardy potential has a nonpositive supersolution if $\lambda>\Lambda_{N, s}$. We only bring it here for completeness. 
Theorem 2.9 Let $s \in(0,1), \lambda>\Lambda_{N, s}$, and $\gamma>0$. Then there is no positive very weaksolution to problem (1).

Proof We argue by contradiction. Let $u$ be a positive very weak solution to problem (1). Then $u$ satisfies

$$
\begin{cases}(-\Delta)^{s} u-\Lambda_{N, s} \frac{u}{|x|^{2 s}}=\left(\lambda-\Lambda_{N, s}\right) \frac{u}{|x|^{2 s}}+g & \text { in } \Omega, \\ u>0 & \text { in } \Omega, \\ u=0 & \text { in }\left(\mathbb{R}^{N} \backslash \Omega\right),\end{cases}
$$

where $g=\frac{\mu}{u^{\gamma}}+f(x)$. Then by using Lemma 2.5 and the positivity of $g$ we have

$$
\left(\left(\lambda-\Lambda_{N, s}\right) \frac{u}{|x|^{2 s}}\right)|x|^{-\beta} \in L^{1}\left(B_{r}(0)\right)
$$

for some $B_{r}(0) \Subset \Omega$. On the other hand, by Lemma 2.4 we have

$$
u(x) \geq C|x|^{-\beta} \quad \text { in } B_{r}(0)
$$

for sufficiently small $r$, where $\beta=\frac{N-2 s}{2}-\alpha$, and $\alpha \in\left[0, \frac{N-2 s}{2}\right)$ is given by the identity

$$
\frac{4^{s} \Gamma^{2}\left(\frac{N+2 s}{4}\right)}{\Gamma^{2}\left(\frac{N-2 s}{4}\right)}=\frac{4^{s} \Gamma\left(\frac{N+2 s+2 \alpha}{4}\right) \Gamma\left(\frac{N+2 s-2 \alpha}{4}\right)}{\Gamma\left(\frac{N-2 s+2 \alpha}{4}\right) \Gamma\left(\frac{N-2 s-2 \alpha}{4}\right)} .
$$

The properties of the gamma function imply $\alpha=0$; see the proof of [42, Lemma 3.3]. Now by combining (11) and (12) we obtain that $|x|^{-N} \in L^{1}\left(B_{r}(0)\right)$, which is a contradiction.

This nonexistence result is strong in the sense that a complete blowup phenomenon occurs. Namely, let $u_{n}$ be the solution to the following approximated problem with $\lambda>$ $\Lambda_{N, s}$, where the Hardy potential is substituted by the bounded weight $\left(|x|^{2 s}+\frac{1}{n}\right)^{-1}$, and the singular nonlinearity is substituted by $\frac{\min \{\mu, n\}}{\left(u_{n}+\frac{1}{n}\right)^{2}}$ :

$$
\begin{cases}(-\Delta)^{s} u_{n}=\lambda \frac{u_{n}}{|x|^{2 s}+\frac{1}{n}}+\frac{\min \{\mu, n\}}{\left(u_{n}+\frac{1}{n}\right)^{\gamma}}+\min \{f, n\} & \text { in } \Omega, \\ u_{n}>0 & \text { in } \Omega, \\ u_{n}=0 & \text { in }\left(\mathbb{R}^{N} \backslash \Omega\right) .\end{cases}
$$

Then $u_{n}\left(x_{0}\right) \rightarrow \infty$ for any $x_{0} \in \Omega$ as $n \rightarrow \infty$.

In the same spirit of Theorem 2.9, we can prove this blowup phenomenon by taking into consideration that any approximating sequence of nonnegative supersolution to the linear problem blows up at any point of $\Omega$ if $\lambda>\Lambda_{N, s}$, as it is proved in [29]. 


\section{Proof of Theorem 2.7 and Theorem 2.8}

We first prove Theorem 2.7. For this purpose, let us consider the following auxiliary problem:

$$
\begin{cases}(-\Delta)^{s} u=\lambda \frac{u}{|x|^{2 s}}+g & \text { in } \Omega, \\ u>0 & \text { in } \Omega, \\ u=0 & \text { in }\left(\mathbb{R}^{N} \backslash \Omega\right),\end{cases}
$$

where $g \in X^{-s}(\Omega)$. The function $u \in X_{0}^{s}(\Omega)$ is a weak energy solution to this problem if $u \equiv 0$ in $\left(\mathbb{R}^{N} \backslash \Omega\right)$ and

$$
\int_{\mathbb{R}^{N}}(-\Delta)^{\frac{s}{2}} u(-\Delta)^{\frac{s}{2}} \phi d x=\lambda \int_{\Omega} \frac{u \phi}{|x|^{2 s}} d x+\langle g, \phi\rangle_{X^{-s}(\Omega), X_{0}^{s}(\Omega)}, \quad \phi \in X_{0}^{s}(\Omega) .
$$

Here $\langle\cdot, \cdot\rangle_{X^{-s}(\Omega), X_{0}^{S}(\Omega)}$ denotes the duality pairing between $X^{-s}(\Omega)$ and $X_{0}^{s}(\Omega)$.

The proof of the following proposition on the existence for (14) can be obtained by using the Hardy inequality and classical variational methods. See, for instance, [43, Sect. 4.6]. Also, the uniqueness of the weak energy solution to (14) follows from the strict monotonicity of the operator $(-\Delta)^{s} u-\lambda \frac{u}{|x|^{2 s}}$ for $0 \leq \lambda<\Lambda_{N, s}$. The strict monotonicity of this operator is a direct consequence of the Hardy inequality.

Proposition 3.1 If $g(x) \in L^{2}(\Omega), s \in(0,1)$, and $0<\lambda<\Lambda_{N, s}$, then there exists a unique positive weak energy solution to (14) in $X_{0}^{s}(\Omega)$.

Before we continue, we need to define the set $\mathcal{C}$ of functions $v \in L^{2}(\Omega)$ such that there exist positive constants $k_{1}$ and $k_{2}$ such that

$$
k_{1} \delta^{s}(x) \leq|x|^{\beta} v(x) \leq k_{2} \delta^{s}(x)
$$

where the constant $\beta$ is given in Lemma 2.4, and $\delta(x)=\operatorname{dist}(x, \partial \Omega), x \in \Omega$, is the distance function from the boundary $\partial \Omega$.

Now for $v \in \mathcal{C}$, define $\Phi(v)=w$, where $w \in X_{0}^{s}(\Omega)$ is the unique solution to the following problem for any fixed $n$ :

$$
\begin{cases}(-\Delta)^{s} w=\lambda \frac{w}{|x|^{2 s}}+\frac{\mu_{n}}{\left(|v|+\frac{1}{n}\right)^{\gamma}}+f_{n}(x) & \text { in } \Omega, \\ w>0 & \text { in } \Omega, \\ w=0 & \text { in }\left(\mathbb{R}^{N} \backslash \Omega\right) .\end{cases}
$$

Here $f_{n}=T_{n}(f)$ and $\mu_{n}=T_{n}(\mu)$ are the truncations at level $n$.

By Lemma 2.4, [29, Theorem 4.1], and a result of [37], it easily follows that $w \in \mathcal{C}$. If we show that $\Phi: \mathcal{C} \rightarrow \mathcal{C}$ has a fixed point $w_{n}$, then $w_{n} \in \mathcal{C}$ will be the weak solution to the following problem in $X_{0}^{s}(\Omega)$ :

$$
\begin{cases}(-\Delta)^{s} w_{n}=\lambda \frac{w_{n}}{|x|^{2 s}}+\frac{\mu_{n}}{\left(w_{n}+\frac{1}{n}\right)^{\gamma}}+f_{n}(x) & \text { in } \Omega, \\ w_{n}>0 & \text { in } \Omega, \\ w_{n}=0 & \text { in }\left(\mathbb{R}^{N} \backslash \Omega\right) .\end{cases}
$$


We apply the Schauder fixed-point theorem (see, e.g., [43, Theorem 3.2.20]). We need to prove that $\Phi$ is continuous and compact and that there exists a bounded convex subset of $\mathcal{C} \subset L^{2}(\Omega)$ that is invariant under $\Phi$.

For continuity, let $v_{k} \rightarrow v$ in $L^{2}(\Omega)$. It is obvious that for each $n$,

$$
\left\|\left(\frac{\mu_{n}}{\left(\left|v_{k}\right|+\frac{1}{n}\right)^{\gamma}}+f_{n}\right)-\left(\frac{\mu_{n}}{\left(|v|+\frac{1}{n}\right)^{\gamma}}+f_{n}\right)\right\|_{L^{2}(\Omega)} \rightarrow 0, \quad k \rightarrow \infty .
$$

Now from the uniqueness of the weak solution to (14) we conclude $\Phi\left(v_{k}\right) \rightarrow \Phi(v)$.

For compactness, we argue as follows. For $v \in \mathcal{C}$, let $w$ be a solution to (15). If $\lambda_{1}^{s}(\Omega)$ is the first eigenvalue of $(-\Delta)^{s}$ in $X_{0}^{s}(\Omega)$ [38, Proposition 9], then we have

$$
\begin{aligned}
\lambda_{1}^{s}(\Omega) \int_{\Omega} w^{2} d x & \leq \int_{\mathbb{R}^{N}}\left|(-\Delta)^{\frac{s}{2}} w\right|^{2} d x \\
& \leq \frac{\Lambda_{N, s}}{\Lambda_{N, s}-\lambda}\left(\int_{\mathbb{R}^{N}}\left|(-\Delta)^{\frac{s}{2}} w\right|^{2}-\lambda \frac{w^{2}}{|x|^{2 s}} d x\right),
\end{aligned}
$$

where in the last inequality we have used the Hardy inequality. Testing (15) with $\phi=w$, we have

$$
\int_{\mathbb{R}^{N}}\left|(-\Delta)^{\frac{s}{2}} w\right|^{2} d x-\lambda \int_{\mathbb{R}^{N}} \frac{w^{2}}{|x|^{2 s}} d x=\int_{\Omega} \frac{\mu_{n}}{\left(|v|+\frac{1}{n}\right)^{\gamma}} w d x+\int_{\Omega} f_{n} w d x .
$$

For the first term on the right-hand side of this equality, we have the estimate

$$
\int_{\Omega} \frac{\mu_{n}}{\left(|v|+\frac{1}{n}\right)^{\gamma}} w d x \leq n^{\gamma} \int_{\Omega} \mu_{n} w d x \leq C_{1}\left(\int_{\Omega}|w|^{2} d x\right)^{\frac{1}{2}}
$$

where in the last inequality, we have used the Hölder inequality. Once more using the Hölder inequality gives $\int_{\Omega} f_{n} w d x \leq C_{2}\left(\int_{\Omega}|w|^{2} d x\right)^{\frac{1}{2}}$ for some $C_{2}>0$. Thus combining this inequality with (17), (18), and (19), we obtain

$$
\lambda_{1}^{s}(\Omega) \int_{\Omega}|w|^{2} d x \leq C_{3}\left(\int_{\Omega}|w|^{2} d x\right)^{\frac{1}{2}},
$$

which implies that $\Phi\left(L^{2}(\Omega)\right)$ is contained in a ball of finite radius in $L^{2}(\Omega)$. Therefore the intersection of this ball with $\mathcal{C}$ is invariant under $\Phi$. Moreover, we have $\int_{\mathbb{R}^{N}}\left|(-\Delta)^{\frac{s}{2}} \Phi(v)\right|^{2} d x=\int_{\mathbb{R}^{N}}\left|(-\Delta)^{\frac{s}{2}} w\right|^{2} d x \leq C_{4}$, which means that $\Phi\left(L^{2}(\Omega)\right)$ is relatively compact in $L^{2}(\Omega)$ by the compactness of embedding (6).

Proposition 3.2 For every $K \Subset \Omega$, there exists $C_{K}>0$ such that $\left\{w_{n}\right\}$, the solutions to (16), satisfy $w_{n}(x) \geq C_{K}$ a.e. in $K$ for each $n$.

Proof Let us consider the following problem:

$$
\begin{cases}(-\Delta)^{s} v_{n}=\frac{\mu_{n}}{\left(v_{n}+\frac{1}{n}\right)^{\gamma}} & \text { in } \Omega, \\ v_{n}>0 & \text { in } \Omega, \\ v_{n}=0 & \text { in }\left(\mathbb{R}^{N} \backslash \Omega\right) .\end{cases}
$$


The existence of a weak solution $v_{n}$ follows from a similar proof to problem (16). In the same way of [17, Lemma 3.2], we can show that $v_{n} \leq v_{n+1}$ a.e. in $\Omega$. Also, for each $K \Subset \Omega$, there exists $C_{K}>0$ such that $v_{1}(x) \geq C_{K}$ a.e. in $K$. Now subtracting the weak formulation of (20) from the weak formulation of (16) and using $\left(w_{n}-v_{n}\right)^{-}$as a test function (see [44, Theorem 20]), we conclude that $w_{n} \geq v_{n}$ a.e. in $\Omega$. Therefore, for every $K \Subset \Omega$, there exists $C_{K}$ such that $w_{n} \geq v_{n} \geq v_{1} \geq C_{k}>0$ a.e. in $K$.

Proposition 3.3 Let $\gamma \geq 1$. Also, let $\left\{w_{n}\right\}_{n=1}^{\infty}$ be the sequence of solutions to (16). Then $\left\{T_{k}^{\frac{\gamma+1}{2}}\left(w_{n}\right)\right\}_{n=1}^{\infty}$ and $\left\{G_{k}\left(w_{n}\right)\right\}_{n=1}^{\infty}$ are bounded in $X_{0}^{s}(\Omega)$, and $\left\{T_{k}\left(w_{n}\right)\right\}_{n=1}^{\infty}$ is bounded in $X_{\mathrm{loc}}^{s}(\Omega)$.

Proof We will follow the proof of [29, Theorem 5.2]. Let $\gamma \geq 1$. Taking $\phi=T_{k}^{\gamma}\left(w_{n}\right)$ as a test function in (16), we obtain

$$
\begin{aligned}
\int_{\mathbb{R}^{N}}(-\Delta)^{\frac{s}{2}} w_{n}(-\Delta)^{\frac{s}{2}} T_{k}^{\gamma}\left(w_{n}\right) d x= & \lambda \int_{\Omega} \frac{w_{n} T_{k}^{\gamma}\left(w_{n}\right)}{|x|^{2 s}} d x \\
& +\int_{\Omega} \frac{\mu_{n}}{\left(w_{n}+\frac{1}{n}\right)^{\gamma}} T_{k}^{\gamma}\left(w_{n}\right) d x+\int_{\Omega} T_{k}^{\gamma}\left(w_{n}\right) f_{n} d x .
\end{aligned}
$$

For the left-hand side, by using (7) and the elementary inequality

$$
\left(s_{1}-s_{2}\right)\left(s_{1}^{\gamma}-s_{2}^{\gamma}\right) \geq \frac{4 \gamma}{(\gamma+1)^{2}}\left(s_{1}^{\frac{\gamma+1}{2}}-s_{2}^{\frac{\gamma+1}{2}}\right)^{2}, \quad \forall s_{1}, s_{2} \geq 0, \gamma>0 \text {, }
$$

we get

$$
\begin{aligned}
& \int_{\mathbb{R}^{N}}(-\Delta)^{\frac{s}{2}} w_{n}(-\Delta)^{\frac{s}{2}} T_{k}^{\gamma}\left(w_{n}\right) d x \\
& \quad=\frac{C_{N, s}}{2} \iint_{D_{\Omega}} \frac{\left(w_{n}(x)-w_{n}(y)\right)\left(T_{k}^{\gamma}\left(w_{n}\right)(x)-T_{k}^{\gamma}\left(w_{n}\right)(y)\right)}{|x-y|^{N+2 s}} d x d y \\
& \quad \geq \frac{2 \gamma C_{N, s}}{(\gamma+1)^{2}} \iint_{D_{\Omega}} \frac{\left|T_{k}^{\frac{\gamma+1}{2}} w_{n}(x)-T_{k}^{\frac{\gamma+1}{2}}\left(w_{n}\right)(y)\right|^{2}}{|x-y|^{N+2 s}} d x d y \\
& \quad \geq C_{0} \int_{\mathbb{R}^{N}}\left|(-\Delta)^{\frac{s}{2}} T_{k}^{\frac{\gamma+1}{2}}\left(w_{n}\right)\right|^{2} d x .
\end{aligned}
$$

For the first term on the right-hand side, we have

$$
\int_{\Omega} \frac{w_{n} T_{k}^{\gamma}\left(w_{n}\right)}{|x|^{2 s}} d x \leq k^{\gamma-1} \int_{\Omega} \frac{w_{n}^{2}}{|x|^{2 s}} d x
$$

For the second term on the right-hand side of (21), note that $\frac{T_{k}^{\gamma}\left(w_{n}\right)}{\left(w_{n}+\frac{1}{n}\right)^{\gamma}} \leq \frac{w_{n}^{\gamma}}{\left(w_{n}+\frac{1}{n}\right)^{\gamma}} \leq 1$. Now we deduce

$$
\int_{\Omega} \frac{\mu_{n}}{\left(w_{n}+\frac{1}{n}\right)^{\gamma}} T_{k}^{\gamma}\left(w_{n}\right) d x \leq \int_{\Omega} \mu_{n} d x \leq\left\|\mu_{n}\right\|_{L^{1}} \leq C_{1} .
$$

Also, for the last term,

$$
\int_{\Omega} T_{k}^{\gamma}\left(w_{n}\right) f_{n} d x \leq k^{\frac{\gamma-1}{2}} \int_{\Omega} T_{k}^{\frac{\gamma+1}{2}}\left(w_{n}\right) f_{n} d x
$$




$$
\begin{aligned}
& =k^{\frac{\gamma-1}{2}}\left\langle f_{n},\left.T_{k}^{\frac{\gamma+1}{2}}\left(w_{n}\right)\right|_{X^{-s}(\Omega), X_{0}^{s}(\Omega)}\right. \\
& \leq k^{\frac{\gamma-1}{2}}\left\langle f,\left.T_{k}^{\frac{\gamma+1}{2}}\left(w_{n}\right)\right|_{X^{-s}(\Omega), X_{0}^{s}(\Omega)}\right. \\
& \leq k^{\frac{\gamma-1}{2}}\|f\|_{X^{-s}(\Omega)}\left\|T_{k}^{\frac{\gamma+1}{2}}\left(w_{n}\right)\right\|_{X_{0}^{s}(\Omega)}=k^{\frac{\gamma-1}{2}} C_{2}\left\|T_{k}^{\frac{\gamma+1}{2}}\left(w_{n}\right)\right\|_{X_{0}^{s}(\Omega)} .
\end{aligned}
$$

Thus from (21), (23), (24), (25), and (26) we obtain

$$
\begin{aligned}
\int_{\mathbb{R}^{N}}\left|(-\Delta)^{\frac{s}{2}} T_{k}^{\frac{\gamma+1}{2}}\left(w_{n}\right)\right|^{2} d x \leq & \frac{\lambda k^{\gamma-1}}{C_{0}} \int_{\mathbb{R}^{N}} \frac{w_{n}^{2}}{|x|^{2 s}} d x \\
& +C_{1}+C_{2} k^{\frac{\gamma-1}{2}}\left\|T_{k}^{\frac{\gamma+1}{2}}\left(w_{n}\right)\right\|_{X_{0}^{s}(\Omega)} .
\end{aligned}
$$

If we show that the term

$$
\int_{\Omega} \frac{w_{n}^{2}}{|x|^{2 s}} d x
$$

is uniformly bounded in $n$, then (27) gives $\left\|T_{k}^{\frac{\gamma+1}{2}}\left(w_{n}\right)\right\|_{X_{0}^{s}(\Omega)}^{2} \leq C_{3}(k)\left(1+\left\|T_{k}^{\frac{\gamma+1}{2}}\left(w_{n}\right)\right\|_{X_{0}^{s}(\Omega)}\right)$, which implies the boundedness of $\left\{T_{k}^{\frac{\gamma+1}{2}}\left(w_{n}\right)\right\}$ in $X_{0}^{s}(\Omega)$.

For proving the boundedness of (28), it suffices to consider $\phi=G_{k}\left(w_{n}\right)$ as a test function in (16) as follows, where $G_{k}(\sigma):=\sigma-T_{k}(\sigma)$ :

$$
\begin{aligned}
\int_{\mathbb{R}^{N}}\left|(-\Delta)^{\frac{s}{2}} G_{k}\left(w_{n}\right)\right|^{2} d x \leq & \lambda \int_{\mathbb{R}^{N}} \frac{w_{n} G_{k}\left(w_{n}\right)}{|x|^{2 s}} d x \\
& +\int_{\Omega} \frac{\mu_{n}}{\left(w_{n}+\frac{1}{n}\right)^{\gamma}} G_{k}\left(w_{n}\right) d x+\int_{\Omega} f_{n} G_{k}\left(w_{n}\right) d x .
\end{aligned}
$$

Note that for the left-hand side, we have used [44, Proposition 3]. To estimate the terms on the right-hand side of this equality uniformly in $n$, we have the following. For the second term on the right-hand side of (29), we have the following estimate uniformly in $n$ :

$$
\int_{\Omega} \frac{\mu_{n}}{\left(w_{n}+\frac{1}{n}\right)^{\gamma}} G_{k}\left(w_{n}\right) d x \leq \frac{1}{k^{\gamma-1}} \int_{\Omega} \mu_{n} \leq C .
$$

For $\int_{\Omega} f_{n} G_{k}\left(w_{n}\right) d x$, we have the following estimate:

$$
\begin{aligned}
\int_{\Omega} f_{n} G_{k}\left(w_{n}\right) d x & =\left\langle f_{n},\left.G_{k}\left(w_{n}\right)\right|_{X^{-s}(\Omega), X_{0}^{s}(\Omega)} \leq\left\langle f, G_{k}\left(w_{n}\right)\right\rangle_{X^{-s}(\Omega), X_{0}^{s}(\Omega)}\right. \\
& \leq C_{1}\left\|G_{k}\left(w_{n}\right)\right\|_{X_{0}^{s}(\Omega)} .
\end{aligned}
$$

For the first term on the right-hand side of (29), we can write

$$
\int_{\mathbb{R}^{N}} \frac{w_{n} G_{k}\left(w_{n}\right)}{|x|^{2 s}} d x=\int_{\mathbb{R}^{N}} \frac{\left|G_{k}\left(w_{n}\right)\right|^{2}}{|x|^{2 s}} d x+k \int_{\mathbb{R}^{N}} \frac{G_{k}\left(w_{n}\right)}{|x|^{2 s}} d x .
$$

For the last term in (30), using the Hölder inequality with exponents $a=2_{s}^{*}$ and $b=\frac{2 N}{N+2 s}<$ $\frac{N}{2 s}$, noting that the integration can be over $\Omega$ because of $w_{n} \equiv 0$ in $\mathbb{R}^{N} \backslash \Omega$, and embedding 
(6), we obtain

$$
\begin{aligned}
\int_{\mathbb{R}^{N}} \frac{G_{k}\left(w_{n}\right)}{|x|^{2 s}} d x & =\int_{\Omega} \frac{G_{k}\left(w_{n}\right)}{|x|^{2 s}} d x \leq\left(\int_{\Omega} \frac{1}{|x|^{2 s b}} d x\right)^{\frac{1}{b}}\left(\int_{\mathbb{R}^{N}}\left|G_{k}\left(w_{n}\right)\right|^{a} d x\right)^{\frac{1}{a}} \\
& \leq C_{2}\left\|G_{k}\left(w_{n}\right)\right\|_{X_{0}^{s}(\Omega)} .
\end{aligned}
$$

Combining the above estimates, from (29) we get

$$
\begin{aligned}
\int_{\mathbb{R}^{N}}\left|(-\Delta)^{\frac{s}{2}} G_{k}\left(w_{n}\right)\right|^{2} d x-\lambda \int_{\mathbb{R}^{N}} \frac{\left|G_{k}\left(w_{n}\right)\right|^{2}}{|x|^{2 s}} d x \leq & k C_{2}\left\|G_{k}\left(w_{n}\right)\right\|_{X_{0}^{s}(\Omega)} \\
& +C+C_{1}\left\|G_{k}\left(w_{n}\right)\right\|_{X_{0}^{s}(\Omega)} .
\end{aligned}
$$

Now the Hardy inequality shows the boundedness of the term $\int_{\mathbb{R}^{N}} \frac{\left|G_{k}\left(w_{n}\right)\right|^{2}}{|x|^{2 s}} d x$, and therefore we obtain the boundedness of (28) by using the fact that $w_{n}^{2} \leq 2\left(T_{k}^{2}\left(w_{n}\right)+G_{k}^{2}\left(w_{n}\right)\right)$, that is,

$$
\begin{aligned}
\int_{\Omega} \frac{w_{n}^{2}}{|x|^{2 s}} d x & \leq 2 \int_{\Omega} \frac{\left|T_{k}\left(w_{n}\right)\right|^{2}}{|x|^{2 s}} d x+2 \int_{\Omega} \frac{\left|G_{k}\left(w_{n}\right)\right|^{2}}{|x|^{2 s}} d x \\
& \leq 2 k^{2} \int_{\Omega} \frac{1}{|x|^{2 s}} d x+2 \int_{\Omega} \frac{\left|G_{k}\left(w_{n}\right)\right|^{2}}{|x|^{2 s}} d x .
\end{aligned}
$$

Moreover, we get the boundedness of $\left\|G_{k}\left(w_{n}\right)\right\|_{X_{0}^{s}(\Omega)}$ uniformly in $n$.

Now we show that $\left\{T_{k}\left(w_{n}\right)\right\}$ is bounded in $X_{\text {loc }}^{s}(\Omega)$. For this purpose, first note that by Proposition 3.2, for any compact set $K \Subset \Omega$, there exists $C(K)>0$ such that

$$
w_{n}(x) \geq w_{1}(x) \geq C(K)>0 \quad \text { a.e. in } K \text {. }
$$

Therefore

$$
T_{k}\left(w_{n}\right) \geq T_{k}\left(w_{1}\right) \geq \tilde{C}:=\min \{k, C(K)\} .
$$

For $(x, y) \in K \times K$, define $\alpha_{n}:=\frac{T_{k}\left(w_{n}\right)(x)}{\tilde{C}}$ and $\beta_{n}:=\frac{T_{k}\left(w_{n}\right)(y)}{\tilde{C}}$. Since $\alpha_{n}, \beta_{n} \geq 1$, we have the following estimate by applying an elementary inequality:

$$
\left(\alpha_{n}-\beta_{n}\right)^{2} \leq\left(\alpha_{n}^{\frac{\gamma+1}{2}}-\beta_{n}^{\frac{\gamma+1}{2}}\right)^{2} .
$$

Now by the definition of $\alpha_{n}$ and $\beta_{n}$ we obtain

$$
\left(T_{k}\left(w_{n}(x)\right)-T_{k}\left(w_{n}(y)\right)\right)^{2} \leq \tilde{C}^{1-\gamma}\left(T_{k}^{\frac{\gamma+1}{2}} w_{n}(x)-T_{k}^{\frac{\gamma+1}{2}} w_{n}(y)\right)^{2} .
$$

Thus we get the boundedness of $\left\{T_{k}\left(w_{n}\right)\right\}_{n=1}^{\infty}$ in $X_{\text {loc }}^{s}(\Omega)$ by using (5) and the boundedness of $\left\{T_{k}^{\frac{\gamma+1}{2}}\left(w_{n}\right)\right\}_{n=1}^{\infty}$ in $X_{0}^{s}(\Omega)$.

Remark 3 In the case $\gamma=1$, since both $\left\{G_{k}\left(w_{n}\right)\right\}_{n=1}^{\infty}$ and $\left\{T_{k}\left(w_{n}\right)\right\}_{n=1}^{\infty}$ are bounded in $X_{0}^{s}(\Omega)$, $\left\{w_{n}\right\}_{n=1}^{\infty}$ is also bounded in $X_{0}^{s}(\Omega)$. 
Remark 4 For the case $0<\gamma<1$, if furthermore we assume $\mu \in L^{\left(\frac{2_{s}^{*}}{1-\gamma}\right)^{\prime}}(\Omega)$, then the sequence $\left\{w_{n}\right\}_{n=1}^{\infty}$ is bounded in $X_{0}^{s}(\Omega)$. Indeed, you just have to keep in mind that

$$
\int_{\Omega} \frac{\mu_{n}}{\left(w_{n}+\frac{1}{n}\right)^{\gamma}} w_{n} d x \leq \int_{\Omega} \mu_{n} w_{n}^{1-\gamma} d x \leq\|\mu\|_{L^{\left(\frac{2_{s}^{*}}{1-\gamma}\right)^{\prime}}(\Omega)}\left\|w_{n}\right\|_{L^{2 *}(\Omega)}^{1-\gamma} \leq C\left\|w_{n}\right\|_{X_{0}^{s}(\Omega)}^{1-\gamma} .
$$

Since the rest of the proof can be obtained proceeding as in the case $\gamma=1$, for brevity, we leave it left to the reader.

Now we are ready to prove Theorem 2.7.

Proof of Theorem 2.7 There exists $u \in X_{\mathrm{loc}}^{s}(\Omega)\left(u \in X_{0}^{s}(\Omega)\right.$ in the case $\left.\gamma \leq 1\right)$ such that, up to a subsequence,

- $w_{n} \rightarrow u$ weakly in $X_{\text {loc }}^{s}(\Omega)$ (weakly in $X_{0}^{s}(\Omega)$ in the case $\gamma \leq 1$ );

- $G_{k}\left(w_{n}\right) \rightarrow G_{k}(u)$ weakly in $X_{0}^{s}(\Omega)$;

- $T_{k}^{\frac{\gamma+1}{2}}\left(w_{n}\right) \rightarrow T_{k}^{\frac{\gamma+1}{2}}(u)$ weakly in $X_{0}^{s}(\Omega)$.

Also, using embedding (6), up to a subsequence, we have

- $w_{n} \rightarrow u$ in $L^{r}(\Omega)$ for any $r \in\left[1,2_{s}^{*}\right)$

- $w_{n}(x) \rightarrow u(x)$ pointwise a.e. in $\Omega$.

Now for every fixed $\phi \in \mathcal{T}(\Omega)$, by the estimates above, we can pass to the limit and obtain

$$
\begin{aligned}
& \int_{\Omega} \frac{w_{n} \phi}{|x|^{2 s}} d x \rightarrow \int_{\Omega} \frac{u \phi}{|x|^{2 s}} d x<+\infty, \\
& \int_{\Omega} \frac{\mu_{n}}{\left(w_{n}+\frac{1}{n}\right)^{\gamma}} \phi d x \rightarrow \int_{\Omega} \frac{\mu \phi}{u^{\gamma}} d x<+\infty, \\
& \int_{\Omega} f_{n} \phi d x \rightarrow \int_{\Omega} f \phi d x .
\end{aligned}
$$

Also, for every $\phi \in \mathcal{T}(\Omega)$, we have

$$
\lim _{n \rightarrow \infty} \int_{\mathbb{R}^{N}}(-\Delta)^{\frac{s}{2}} w_{n}(-\Delta)^{\frac{s}{2}} \phi d x=\lim _{n \rightarrow \infty} \int_{\mathbb{R}^{N}} w_{n}(-\Delta)^{s} \phi d x=\int_{\mathbb{R}^{N}} u(-\Delta)^{s} \phi d x .
$$

Since for every $K \Subset \Omega$, there exists $C_{K}>0$ such that $w_{n}(x) \geq C_{K}$ a.e. in $K$ and also $w_{n} \equiv 0$ in $\left(\mathbb{R}^{N} \backslash \Omega\right)$, and because of $w_{n}(x) \rightarrow u(x)$ a.e. in $\Omega, u$ is a weak solution to problem (1).

Finally, note that if we take $\gamma$ such that $\frac{4 \gamma}{(\gamma+1)^{2}}>\frac{\lambda}{\Lambda_{N, s}}$, then by testing (16) with $w_{n}^{\gamma}$ and using inequality (22) together with Hardy inequality, it easily follows that $u^{\frac{\gamma+1}{2}} \in X_{0}^{s}(\Omega)$.

By now, in Theorem 2.9, we have shown that for $\lambda>\Lambda_{N, s}$, there is no positive solution to problem (1). Also, in Theorem 2.7, we have proved the existence of a positive solution for $\lambda<\Lambda_{N, s}$. The following remark for $\lambda=\Lambda_{N, s}$ may be interesting.

Remark 5 In the borderline case $\lambda=\Lambda_{N, s}$, by invoking the improved version of Hardy inequality [45] we can define the space $H(\Omega)$ as the completion of $C_{0}^{\infty}(\Omega)$ with respect to the norm

$$
\|\phi\|_{H(\Omega)}:=\left(\int_{\mathbb{R}^{N}}\left|(-\Delta)^{\frac{s}{2}} \phi\right|^{2} d x-\Lambda_{N, s} \int_{\Omega} \frac{\phi^{2}}{|x|^{2 s}} d x\right)^{\frac{1}{2}} .
$$


It is known that $X_{0}^{s}(\Omega) \subsetneq H(\Omega) \subsetneq X_{0}^{s, q}(\Omega)$ for all $q<2$. By invoking the classical variational methods in the space $H(\Omega)$ and the same techniques used above we can obtain a similar existence result in this new function space. See [45, Remark 1] and also [40] for the details.

Now, in the spirit of [29, Theorem 4.10], we prove Theorem 2.8, which gives a necessary and sufficient condition for the existence of a solution to (1).

Proof of Theorem 2.8 Let $u$ be a weak solution to problem (1), and let $\phi_{n} \in \mathcal{T}(\Omega)$ be weak energy solutions to the following problems:

$$
\begin{cases}(-\Delta)^{s} \phi_{n}=\lambda \frac{\phi_{n-1}}{|x|^{2 s}+\frac{1}{n}}+1 & \text { in } \Omega, \\ \phi_{n}>0 & \text { in } \Omega, \\ \phi_{n}=0 & \text { in }\left(\mathbb{R}^{N} \backslash \Omega\right),\end{cases}
$$

where the iteration starts with

$$
\begin{cases}(-\Delta)^{s} \phi_{0}=1 & \text { in } \Omega, \\ \phi_{0}>0 & \text { in } \Omega, \\ \phi_{0}=0 & \text { in }\left(\mathbb{R}^{N} \backslash \Omega\right) .\end{cases}
$$

The comparison principle for fractional Laplacian operator implies that $\phi_{0} \leq \phi_{1} \leq \cdots \leq$ $\phi_{n-1} \leq \phi_{n} \leq \phi$, where $\phi:=\lim _{n \rightarrow \infty} \phi_{n}$ is the weak energy solution to

$$
\begin{cases}(-\Delta)^{s} \phi=\lambda \frac{\phi}{|x|^{2 s}}+1 & \text { in } \Omega, \\ \phi>0 & \text { in } \Omega, \\ \phi=0 & \text { in }\left(\mathbb{R}^{N} \backslash \Omega\right) .\end{cases}
$$

Using $\phi_{n}$ as a test function in (1) yields

$$
\int_{\mathbb{R}^{N}} u(-\Delta)^{s} \phi_{n} d x=\lambda \int_{\Omega} \frac{u \phi_{n}}{|x|^{2 s}} d x+\int_{\Omega} \frac{\mu \phi_{n}}{u^{\gamma}} d x+\int_{\Omega} f \phi_{n} d x .
$$

On the other hand, by the definition $\phi_{n}$ we have

$$
\int_{\mathbb{R}^{N}} u(-\Delta)^{s} \phi_{n} d x=\lambda \int_{\Omega} \frac{u \phi_{n-1}}{|x|^{2 s}+\frac{1}{n}} d x+\int_{\Omega} u d x .
$$

Combining (32) and (33) and noticing that $\frac{\phi_{n-1}}{|x|^{2 s}+\frac{1}{n}} \leq \frac{\phi_{n}}{|x|^{2}}$, we get

$$
\int_{\Omega} f \phi_{n} d x \leq \int_{\Omega} u d x=C
$$

Therefore the sequence $\left\{f \phi_{n}\right\}$ is uniformly bounded in $L^{1}(\Omega)$. Also, since $\left\{f \phi_{n}\right\}$ is increasing, applying the monotone convergence theorem and invoking Lemma 2.4, we obtain

$$
C_{1} \int_{B_{r}(0)}|x|^{-\beta} f d x \leq \int_{\Omega} f \phi d x \leq C .
$$


Also, from Remark 1 it follows that

$$
\int_{\Omega} \frac{\mu}{\delta^{s(\gamma-1)}} d x<+\infty
$$

Now assume that

$$
\int_{B_{r}(0)}|x|^{-\beta} f d x \leq C \quad \text { for some } r \text { and } B_{r}(0) \Subset \Omega
$$

and

$$
\int_{\Omega} \frac{\mu}{\delta^{s(\gamma-1)}} d x<+\infty
$$

Let $u_{n} \in X_{0}^{s}(\Omega)$ be weak energy solutions to the problems

$$
\begin{cases}(-\Delta)^{s} u_{n}=\lambda \frac{u_{n-1}}{|x|^{2 s}+\frac{1}{n}}+\frac{\mu}{\left(u_{n-1}+\frac{1}{n}\right)^{\gamma}}+f_{n} & \text { in } \Omega, \\ u_{n}>0 & \text { in } \Omega, \\ u_{n}=0 & \text { in }\left(\mathbb{R}^{N} \backslash \Omega\right),\end{cases}
$$

where

$$
\begin{cases}(-\Delta)^{s} u_{0}=f_{1} & \text { in } \Omega, \\ u_{0}>0 & \text { in } \Omega, \\ u_{0}=0 & \text { in }\left(\mathbb{R}^{N} \backslash \Omega\right) .\end{cases}
$$

Here $f_{n}=T_{n}(f)$. Again we have $u_{0} \leq u_{1} \leq \cdots \leq u_{n-1} \leq u_{n}$ in $\mathbb{R}^{N}$. Using $\phi \in X_{0}^{s}(\Omega)$, the solution to (31), as a test function in (36), we obtain

$$
\int_{\mathbb{R}^{N}} u_{n}(-\Delta)^{s} \phi d x=\lambda \int_{\Omega} \frac{u_{n-1} \phi}{|x|^{2 s}+\frac{1}{n}} d x+\int_{\Omega} \frac{\mu \phi}{\left(u_{n-1}+\frac{1}{n}\right)^{\gamma}} d x+\int_{\Omega} f_{n} \phi d x
$$

On the other hand, using $u_{n}$ as a test function in the weak formulation of (31), we get

$$
\int_{\mathbb{R}^{N}} u_{n}(-\Delta)^{s} \phi d x=\lambda \int_{\Omega} \frac{u_{n} \phi}{|x|^{2 s}} d x+\int_{\Omega} u_{n} d x
$$

From (37) and (38), using Lemma 2.4 together with (34) and (35), we obtain

$$
\begin{aligned}
\int_{\Omega} u_{n} d x & \leq \int_{\Omega} f_{n} \phi d x+\int_{\Omega} \frac{\mu \phi}{\left(u_{n-1}+\frac{1}{n}\right)^{\gamma}} d x \leq \int_{\Omega} f \phi d x+\int_{\Omega} \frac{\mu \phi}{u_{0}^{\gamma}} d x \\
& \leq C_{1} \int_{\Omega} f|x|^{-\beta} d x+c_{1} c^{-\gamma} \int_{\Omega} \frac{\mu}{\delta^{s(\gamma-1)}} d x \\
& \leq C .
\end{aligned}
$$

Note that in the last inequality, we have used $u_{0} \geq c \delta^{s}$ and $\phi \sim c_{1} \delta^{s}$ near the boundary $\partial \Omega$ for some $c_{1}>0$, since $\phi$ is a solution to (31). This follows by a result of [37] together with the comparison principle for the fractional Laplacian. 
Since $u_{n}$ is increasing and also uniformly bounded in $L^{1}(\Omega)$, by the monotone convergence theorem we conclude that $u:=\lim _{n \rightarrow \infty} u_{n}$ is a function in $L^{1}(\Omega)$. We want to show that $u$ is a weak solution to problem (1). For this purpose, let $\psi \in X_{0}^{s}(\Omega) \cap L^{\infty}(\Omega)$ be the unique positive weak energy solution to

$$
\begin{cases}(-\Delta)^{s} \psi=1 & \text { in } \Omega, \\ \psi=0 & \text { in }\left(\mathbb{R}^{N} \backslash \Omega\right) .\end{cases}
$$

Using $\psi$ as a test function in (36) and noting that $\psi \sim \delta^{s}$, from (39) we get

$$
\lambda \int_{\Omega} \frac{u_{n-1}}{|x|^{2 s}+\frac{1}{n}} \delta^{s} d x+\int_{\Omega} \frac{\mu}{\left(u_{n-1}+\frac{1}{n}\right)^{\gamma}} \delta^{s} d x \leq C_{2} \int_{\Omega} u_{n} d x \leq C_{2} C .
$$

Thus by applying the monotone convergence theorem we get

$$
\frac{u_{n-1}}{|x|^{2 s}+\frac{1}{n}}+f_{n} \nearrow \frac{u}{|x|^{2 s}}+f, \quad \text { in } L^{1}\left(\Omega, \delta^{s} d x\right)
$$

Also, since

$$
\left|\frac{\mu}{\left(u_{n-1}+\frac{1}{n}\right)^{\gamma}} \delta^{s}\right| \leq\left|\frac{\mu}{u_{0}^{\gamma}} \delta^{s}\right| \leq \frac{\mu}{\delta^{s(\gamma-1)}} \in L^{1}(\Omega),
$$

by the dominated convergence theorem we have

$$
\frac{\mu}{\left(u_{n-1}+\frac{1}{n}\right)^{\gamma}} \rightarrow \frac{\mu}{u^{\gamma}} \quad \text { in } L^{1}\left(\Omega, \delta^{s} d x\right)
$$

Therefore $u$ satisfies equation (1) in the following weak sense:

$$
\int_{\mathbb{R}^{N}} u(-\Delta)^{s} \phi d x=\lambda \int_{\Omega} \frac{u \phi}{|x|^{2 s}} d x+\int_{\Omega} \frac{\mu \phi}{u^{\gamma}} d x+\int_{\Omega} f \phi d x, \quad \forall \phi \in \mathcal{T}(\Omega) .
$$

Testing $T_{k}\left(u_{n}\right)$ in (36) and using (35), we can show that $T_{k}\left(u_{n}\right) \rightarrow T_{k}(u)$ weakly in $X_{0}^{s}(\Omega)$ (similarly to the arguments in the proof of Proposition 3.3). Moreover, since $\lambda \frac{u_{n-1}}{|x|^{2 s}+\frac{1}{n}}+$ $\frac{\mu}{\left(u_{n-1}+\frac{1}{n}\right)^{\gamma}}+f_{n}$ converges strongly in $L^{1}\left(\Omega, \delta^{s} d x\right)$, by mimicking the proofs of [46, Proposition 2.3] and [44, Theorem 23] (or directly by adapting the Green operator's viewpoint of the Laplacian case [47, Theorem 1.2.2]), we obtain

- $u \in L^{p}(\Omega)$ for all $p \in\left[1, \frac{N}{N-2 s}\right)$;

- $(-\Delta)^{\frac{s}{2}} u \in L^{p}(\Omega)$ for all $p \in\left[1, \frac{N}{N-s}\right)$.

Since $N>2 s$, we have $\frac{N}{N-s}<2$. Now by invoking Theorem 5 and Proposition 10 in Chap. 5 of [48] we get that $u \in X_{0}^{s_{1}, p}(\Omega)$ for all $s_{1}<s$ and $p<\frac{N}{N-s}$. $\left(\operatorname{In}[48], X_{0}^{s, p}(\Omega)\right.$ reads as $\Lambda_{s}^{p, p}\left(\mathbb{R}^{N}\right)$, and $\mathcal{L}_{s}^{p}\left(\mathbb{R}^{N}\right)$ denotes the space of Bessel potentials; see [48, Sect. 3.2].)

\section{Some uniqueness results and the rate of the growth of solutions}

In this section, we give some uniqueness results. Also, under some summability assumptions on the data of $\mu$ and $f$, we find the growth rate of solutions.

First, for the particular case $\mu \equiv 1$, by studying the behavior of solutions near the boundary we discuss the uniqueness of solutions to problem (1). 
Proposition 4.1 If $\mu \equiv 1$, then the solution obtained to problem (1) in Theorem 2.7 behaves as follows:

$$
\begin{cases}k_{1} \delta^{s}(x) \leq|x|^{\beta} u(x), & 0<\gamma<1 \\ k_{1} \delta^{s}(x)\left(\ln \left(\frac{r}{\delta^{s}(x)}\right)\right)^{\frac{1}{2}} \leq|x|^{\beta} u(x), & \gamma=1, \\ k_{1} \delta^{\frac{2 s}{\gamma+1}}(x) \leq|x|^{\beta} u(x), & \gamma>1,\end{cases}
$$

for any $x \in \Omega$ and some $k_{1}>0$, where $r>\operatorname{diam}(\Omega)$. Here $\beta$ is as defined in Lemma 2.4.

Proof First, notice that by Lemma 2.4 there exists a constant $C_{1}>0$ such that

$$
|x|^{\beta} u(x) \geq C_{1} \quad \text { in } B_{\epsilon}(0) .
$$

Now let $w$ be a weak energy solution to the problem

$$
\begin{cases}(-\Delta)^{s} w=\frac{1}{w^{\gamma}} & \text { in } \Omega, \\ w>0 & \text { in } \Omega, \\ w=0 & \text { in }\left(\mathbb{R}^{N} \backslash \Omega\right) .\end{cases}
$$

By [30, Theorem 2.9] or [49, Theorem 1.2] $w$ satisfies:

$$
\begin{cases}k_{1} \delta^{s}(x) \leq w(x) \leq k_{2} \delta^{s}(x), & 0<\gamma<1, \\ k_{1} \delta^{s}(x)\left(\ln \left(\frac{r}{\delta^{s}(x)}\right)\right)^{\frac{1}{2}} \leq w(x) \leq k_{1} \delta^{s}(x)\left(\ln \left(\frac{r}{\delta^{s}(x)}\right)\right)^{\frac{1}{2}}, & \gamma=1, \\ k_{1} \delta^{\frac{2 s}{\gamma+1}}(x) \leq w(x) \leq k_{2} \delta^{\frac{2 s}{\gamma+1}}(x), & \gamma>1,\end{cases}
$$

for some $k_{1}, k_{2}>0, r>\operatorname{diam}(\Omega)$, and any $x \in \Omega$. By the comparison principle for the fractional Laplacian operator (see, e.g., [5, Proposition 2.17]) we obtain $u(x) \geq w(x)$, which, together with (41) and (42), gives (40).

Remark 6 Note that by using the estimates in Proposition 4.1 and applying the Hölder and fractional Hardy-Sobolev inequalities (and convexity of $\Omega$ only for $0<s<\frac{1}{2}$ ) [50, Theorem 1.1] we get

- For $0<\gamma<1$,

$$
\begin{aligned}
\left|\int_{\Omega} \frac{\phi}{u^{\gamma}} d x\right| & \leq k_{1}^{-\gamma} \int_{\Omega} \frac{|x|^{\beta \gamma}|\phi|}{\delta^{s \gamma}} d x \leq C\left(\int_{\Omega} \frac{\phi^{2}}{\delta^{2 s \gamma}} d x\right)^{\frac{1}{2}} \leq C_{1}\|\phi\|_{X_{0}^{s \gamma}(\Omega)} \\
& \leq C_{2}\|\phi\|_{X_{0}^{s}(\Omega)}
\end{aligned}
$$

where in the last inequality, we used the continuous embedding of $X_{0}^{s_{2}}(\Omega)$ into $X_{0}^{s_{1}}(\Omega)$ for $s_{1}<s_{2}$,

- For $\gamma=1$,

$$
\begin{aligned}
\left|\int_{\Omega} \frac{\phi}{u} d x\right| & \leq k_{1}^{-1} \int_{\Omega} \frac{|x|^{\beta}|\phi|}{\delta^{s}(x)\left(\ln \left(\frac{r}{\delta^{s}(x)}\right)\right)^{\frac{1}{2}}} d x \\
& \leq C\left(\int_{\Omega} \frac{1}{\left|\ln \left(\frac{r}{\delta^{s}(x)}\right)\right|} d x\right)^{\frac{1}{2}}\left(\int_{\Omega} \frac{\phi^{2}}{\delta^{2 s}} d x\right)^{\frac{1}{2}} \leq C_{1}\|\phi\|_{X_{0}^{s}(\Omega)}
\end{aligned}
$$


- For $\gamma>1$,

$$
\begin{aligned}
\left|\int_{\Omega} \frac{\phi}{u^{\gamma}} d x\right| & \leq k_{1}^{-\gamma} \int_{\Omega} \frac{|x|^{\beta \gamma}|\phi|}{\delta^{\frac{2 s \gamma}{\gamma+1}}} d x \leq C\left(\int_{\Omega} \frac{1}{\delta^{2 s \frac{\gamma-1}{\gamma+1}}} d x\right)^{\frac{1}{2}}\left(\int_{\Omega} \frac{\phi^{2}}{\delta^{2 s}} d x\right)^{\frac{1}{2}} \\
& \leq C_{1}\left(\int_{\Omega} \frac{1}{\delta^{2 s^{\gamma+1}}} d x\right)^{\frac{1}{2}}\|\phi\|_{X_{0}^{s}(\Omega)} .
\end{aligned}
$$

If, in addition, $2 s(\gamma-1)<\gamma+1$, then

$$
\int_{\Omega} \frac{\phi}{u^{\gamma}} d x \leq C_{2}\|\phi\|_{X_{0}^{s}(\Omega)}
$$

For general domains with some boundary regularity, the fractional Hardy-Sobolev inequality is proved for $s \in\left[\frac{1}{2}, 1\right)$; see [51-53]. However, in [50] the authors proved the fractional Hardy-Sobolev inequality for any $s \in(0,1)$ by using the facts that the domain is a convex set and its distance from the boundary is a superharmonic function.

Let $u_{1}$ and $u_{2}$ be solutions in $X_{\mathrm{loc}}^{s}(\Omega)$ to problem (1) in the particular case where $\mu \equiv 1$ and either $0<\gamma \leq 1$ or $\gamma>1$ with $2 s(\gamma-1)<\gamma+1$ and define $w=u_{1}-u_{2}$. Then we have

$$
\int_{\mathbb{R}^{N}} w(-\Delta)^{s} \phi d x=\lambda \int_{\Omega} \frac{w \phi}{|x|^{2 s}} d x+\int_{\Omega} \frac{\phi}{u_{1}^{\gamma}}-\frac{\phi}{u_{2}^{\gamma}} d x, \quad \forall \phi \in \mathcal{T}(\Omega) .
$$

The fractional Hardy-Sobolev inequality and a density argument show that equality (43) holds for all $\phi \in X_{0}^{s}(\Omega)$; see Remark 6 . This means that $w \in X_{0}^{s}(\Omega)$. Now by using $w^{-}$as a test function in (43) and applying the ardy inequality we deduce that $w^{-} \equiv 0$. So we arrive at the conclusion that $u_{1} \geq u_{2}$. A similar argument shows that $u_{1} \leq u_{2}$. Therefore $u_{1}=u_{2}$, and the uniqueness follows.

Remark 7 The assumption $\mu \equiv 1$ is taken for simplification. However, the above argument works for any $\mu \geq m$ for some positive constant $m$ such that

$$
\begin{cases}\int_{\Omega} \mu^{2} \delta^{2 s(1-\gamma)} d x<+\infty & 0<\gamma<1 \\ \int_{\Omega} \frac{\mu^{2}}{\ln \left(\frac{r}{\delta^{s}}\right) \mid} d x<+\infty & \gamma=1 \\ \int_{\Omega} \mu^{2} \delta^{2 s \frac{1-\gamma}{1+\gamma}} d x<+\infty & \gamma>1, \text { and } \gamma(2 s-1)<(2 s+1)\end{cases}
$$

For a further discussion, see [30, Theorem 5.2] on a Brezis-Oswald-type uniqueness result.

Once again, because of the interest in uniqueness, we have another definition of solutions to (1). In fact, we would like to consider the entropy solution. The motivation of the definition comes from the works $[54,55]$. In what follows, we consider $0<\gamma \leq 1$.

Definition 4.2 Let $0 \leq \mu, f \in L^{1}(\Omega)$ and $0<\gamma \leq 1$. We say that $u$ is an entropy solution to (1) if

- for every $K \Subset \Omega$, there exists $C_{K}>0$ such that $u(x) \geq C_{K}$ in $K$ and also $u \equiv 0$ in $\mathbb{R}^{N} \backslash \Omega$; 
- $T_{k}(u) \in X_{0}^{s}(\Omega)$ for every $k$, and $u$ satisfies the following family of inequalities:

$$
\begin{aligned}
\int_{\{|u-\phi|<k\}}(-\Delta)^{\frac{s}{2}} u(-\Delta)^{\frac{s}{2}}(u-\phi) d x \leq & \lambda \int_{\Omega} \frac{u T_{k}(u-\phi)}{|x|^{2 s}} d x+\int_{\Omega} u^{-\gamma} \mu T_{k}(u-\phi) d x \\
& +\int_{\Omega} f T_{k}(u-\phi) d x
\end{aligned}
$$

for all $k$ and all $\phi \in X_{0}^{s}(\Omega) \cap L^{\infty}(\Omega)$, together with the assumption that the second term on the right-hand side of the inequality is finite for all $\phi \in X_{0}^{s}(\Omega) \cap L^{\infty}(\Omega)$. The well-posedness of this term will be clear after the construction of entropy solution.

Let $u$ and $v$ be entropy solutions. Testing $u$ with $\phi=T_{h}(v)$ and $v$ with $\phi=T_{h}(u)$ in the weak formulation of entropy inequalities, we have

$$
\begin{aligned}
& \int_{\left\{\left|u-T_{h}(v)\right|<k\right\}}(-\Delta)^{\frac{s}{2}} u(-\Delta)^{\frac{s}{2}}\left(u-T_{h}(v)\right) d x-\lambda \int_{\Omega} \frac{u T_{k}\left(u-T_{h}(v)\right)}{|x|^{2 s}} d x \\
& \leq \int_{\Omega} \frac{\mu T_{k}\left(u-T_{h}(v)\right)}{u^{\gamma}} d x+\int_{\Omega} f T_{k}\left(u-T_{h}(v)\right) d x
\end{aligned}
$$

and

$$
\begin{aligned}
& \int_{\left\{\left|v-T_{h}(u)\right|<k\right\}}(-\Delta)^{\frac{s}{2}} v(-\Delta)^{\frac{s}{2}}\left(v-T_{h}(u)\right) d x-\lambda \int_{\Omega} \frac{v T_{k}\left(v-T_{h}(u)\right)}{|x|^{2 s}} d x \\
& \leq \int_{\Omega} \frac{\mu T_{k}\left(v-T_{h}(u)\right)}{v^{\gamma}} d x+\int_{\Omega} f T_{k}\left(v-T_{h}(u)\right) d x .
\end{aligned}
$$

Summing the left-hand sides of (44) and (45) and restricting them to

$$
A_{0}^{h}=\{x \in \Omega:|u-v|<k,|u|<h,|v|<h\},
$$

we have the following estimate by using the Hardy inequality:

$$
\int_{A_{0}^{h}}\left|(-\Delta)^{\frac{s}{2}}(u-v)\right|^{2} d x-\lambda \int_{A_{0}^{h}} \frac{(u-v)^{2}}{|x|^{2 s}} d x \geq \frac{\Lambda_{N, s}-\lambda}{\Lambda_{N, s}} \int_{A_{0}^{h}}\left|(-\Delta)^{\frac{s}{2}}(u-v)\right|^{2} d x .
$$

Also, summing the right-hand sides of (44) and (45) when restricted to $A_{0}^{h}$ gives

$$
\int_{A_{0}^{h}}(u-v)\left(u^{-\gamma}-v^{-\gamma}\right) \mu d x \leq 0
$$

Now consider the set $A_{1}^{h}=\left\{x \in \Omega:\left|u-T_{h}(v)\right|<k,|v| \geq h\right\}$. When restricted to $A_{1}^{h}$, for the left-hand side of (44), we have:

$$
\begin{aligned}
\int_{A_{1}^{h}}\left|(-\Delta)^{\frac{s}{2}} u\right|^{2} d x-\lambda \int_{A_{1}^{h}} \frac{u(u-h)}{|x|^{2 s}} d x & \geq \int_{A_{1}^{h}}\left|(-\Delta)^{\frac{s}{2}} u\right|^{2} d x-\lambda \int_{A_{1}^{h}} \frac{u^{2}}{|x|^{2 s}} d x \\
& \geq \frac{\Lambda_{N, s}-\lambda}{\Lambda_{N, s}} \int_{A_{1}^{h}}\left|(-\Delta)^{\frac{s}{2}} u\right|^{2} d x \geq 0 .
\end{aligned}
$$


On the other hand, when restricted to $A_{1}^{h}$, the right-hand side of (44) is

$$
\int_{A_{1}^{h}} u^{-\gamma}(u-h) \mu d x+\int_{A_{1}^{h}} f(u-h) d x
$$

which goes to zero as $h \rightarrow \infty$.

Finally, on the remaining set $A_{2}^{h}=\left\{x \in \Omega:\left|u-T_{h}(v)\right|<k,|v|<h,|u| \geq h\right\}$, the left-hand side of (44) is

$$
\int_{A_{2}^{h}}(-\Delta)^{\frac{s}{2}} u(-\Delta)^{\frac{s}{2}}(u-v) d x-\lambda \int_{A_{2}^{h}} \frac{u(u-v)}{|x|^{2 s}} d x
$$

which goes to zero as $h \rightarrow \infty$.

The right-hand side of (44), when restricted to $A_{2}^{h}$, is

$$
\int_{A_{2}^{h}} u^{-\gamma}(u-v) \mu d x+\int_{A_{2}^{h}} f(x)(u-v) d x,
$$

which also goes to zero as $h \rightarrow \infty$.

Similarly, we can estimate the left-hand side of (45) on the sets $B_{1}^{h}=\left\{x \in \Omega:\left|v-T_{h}(u)\right|<\right.$ $k,|u| \geq h\}$ and $B_{2}^{h}=\left\{x \in \Omega:\left|v-T_{h}(u)\right|<k,|u|<h,|v| \geq h\right\}$ and find that

$$
\int_{B_{1}^{h}}\left|(-\Delta)^{\frac{s}{2}} v\right|^{2} d x-\lambda \int_{B_{1}^{h}} \frac{v(v-h)}{|x|^{2 s}} d x \geq \frac{\Lambda_{N, s}-\lambda}{\Lambda_{N, s}} \int_{B_{1}^{h}}\left|(-\Delta)^{\frac{s}{2}} v\right|^{2} d x \geq 0
$$

and

$$
\int_{B_{2}^{h}}(-\Delta)^{\frac{s}{2}} v(-\Delta)^{\frac{s}{2}}(v-u) d x-\lambda \int_{B_{2}^{h}} \frac{v(v-u)}{|x|^{2 s}} d x \rightarrow 0 \quad \text { as } h \rightarrow 0 .
$$

On the other hand, for the right-hand side of (45) on the sets $B_{1}^{h}=\left\{x \in \Omega:\left|v-T_{h}(u)\right|<\right.$ $k,|u| \geq h\}$ and $B_{2}^{h}=\left\{x \in \Omega:\left|v-T_{h}(u)\right|<k,|u|<h,|v| \geq h\right\}$, we have

$$
\int_{B_{1}^{h}} v^{-\gamma}(v-h) \mu d x+\int_{B_{1}^{h}} f(v-h) d x \rightarrow 0 \quad \text { as } h \rightarrow 0
$$

and

$$
\int_{B_{2}^{h}} v^{-\gamma}(v-u) \mu d x+\int_{B_{2}^{h}} f(v-u) d x \rightarrow 0 \quad \text { as } h \rightarrow 0 .
$$

Putting all estimates (46), (47), (48), (49), (50), (51), (52), (53), (54), and (55) together, we obtain

$$
\int_{A_{0}^{h}}\left|(-\Delta)^{\frac{s}{2}}(u-v)\right|^{2} d x \leq \mathrm{o}(h) \quad \text { as } h \rightarrow 0 .
$$

Now, since $A_{0}^{h}$ goes to $\{|u-v|<k\}$ as $h \rightarrow 0$, we have

$$
\int_{\{|u-v|<k\}}\left|(-\Delta)^{\frac{s}{2}}(u-v)\right|^{2} d x \leq 0, \quad \forall k .
$$

Therefore $u \equiv v$, and the uniqueness is proved. 
Now we construct an entropy solution for the case where $0<\gamma \leq 1, \mu \in L^{\left(\frac{2 *}{1-\gamma}\right)^{\prime}}(\Omega) \cap$ $L^{2}(\Omega)$, and a datum of $f \in L^{1}(\Omega)$ satisfying the integrability condition (10). Let consider the following approximating problems:

$$
\begin{cases}(-\Delta)^{s} u_{n}=\lambda \frac{u_{n}}{|x|^{2 s}}+\frac{\mu_{n}}{\left(u_{n}+\frac{1}{n}\right)^{\gamma}}+f_{n} & \text { in } \Omega, \\ u_{n}>0 & \text { in } \Omega, \\ u_{n}=0 & \text { in }\left(\mathbb{R}^{N} \backslash \Omega\right) .\end{cases}
$$

Here $\mu_{n}=T_{n}(\mu)$ and $f_{n}=T_{n}(f)$. The increasing behavior of $\mu_{n}\left(u_{n}+\frac{1}{n}\right)^{-\gamma}+f_{n}$ and the monotonicity of the operator $(-\Delta)^{s} u-\lambda \frac{u}{|x|^{2 s}}$ ensures the existence of an increasing sequence of solutions to problems (56). Testing (56) with $T_{k}\left(u_{n}-\phi\right)$ implies that $\left\{T_{k}\left(u_{n}-\phi\right)\right\}_{n=1}^{\infty}$ is a bounded sequence in $X_{0}^{s}(\Omega)$ for each fixed $k$ and each fixed $\phi \in X_{0}^{s}(\Omega) \cap L^{\infty}(\Omega)$. Therefore, up to a subsequence, $T_{k}\left(u_{n}-\phi\right) \rightarrow T_{k}(u-\phi)$ weakly in $X_{0}^{s}(\Omega)$ as $n \rightarrow \infty$, where $u$ is the weak solution to (1) with $\mu \in L^{\left(\frac{22_{s}^{*}}{1-\gamma}\right)^{\prime}}(\Omega) \cap L^{2}(\Omega)$. Also, since $\left\{T_{k}\left(u_{n}-\phi\right)\right\}_{n=1}^{\infty}$ is an increasing sequence of nonnegative functions, the strict monotonicity of $(-\Delta)^{s}$ again implies that $T_{k}\left(u_{n}-\phi\right) \rightarrow T_{k}(u-\phi)$ strongly in $X_{0}^{s}(\Omega)$ (see, e.g., [29, Lemma 2.18] for this compactness result). Now, using $T_{k}\left(u_{n}-\phi\right)$ as a test function in (56) and noting that

$$
\begin{aligned}
\int_{\Omega}\left|\frac{\mu_{n} T_{k}\left(u_{n}-\phi\right)}{\left(u_{n}+\frac{1}{n}\right)^{\gamma}}\right| d x & \leq \int_{\Omega} \frac{\mu_{n}\left|T_{k}\left(u_{n}-\phi\right)\right|}{u_{1}^{\gamma}} d x \\
& \leq\|\mu\|_{L^{2}(\Omega)} \int_{\Omega} \frac{T_{k}^{2}\left(u_{n}-\phi\right)}{\delta^{2 s \gamma}} d x \\
& \leq C_{1}\left\|\left|T_{k}\left(u_{n}-\phi\right)\right|\right\|_{X_{0}^{s \gamma}(\Omega)} \\
& \leq C_{2}\left\|T_{k}\left(u_{n}-\phi\right)\right\|_{X_{0}^{s}(\Omega)} \leq C_{3}<+\infty \quad \text { uniformly in } n
\end{aligned}
$$

(because $u_{1} \sim c \delta^{s}$ near the boundary and applying the Hölder and fractional HardySobolev inequalities), we may pass to the limit and find an entropy solution even with the equalities instead of the inequalities in Definition 4.2. Note that from the above estimate by Fatou's lemma we deduce

$$
\int_{\Omega} \frac{T_{k}(u-\phi) \mu}{u^{\gamma}} d x<+\infty
$$

for all $\phi \in X_{0}^{s}(\Omega) \cap L^{\infty}(\Omega)$ and $k>0$.

We end this section by a Calderón-Zygmund-type property of solutions to problem (1). See [41] for this property in the local case without the presence of singular nonlinearity and [15] for the case without the Hardy potential.

As mentioned before in Lemma 2.4, any supersolution to (1) is unbounded, that is, $u(x) \gtrsim|x|^{-\beta}$ in a neighborhood of the origin. Now we have the following result, which says that this rate is precisely the rate of growth of $u$ for the regular data of $\mu$ and $f$.

Theorem 4.3 Let $0 \leq \mu, f \in L^{m}(\Omega), m>\frac{N}{2 s}$, and let $u \in X_{0}^{s}(\Omega)$ be the weak energy solution to (1) with $0<\lambda<\Lambda_{N, s}$. Then $u(x) \leq C|x|^{-\beta}$ a.e. in $\Omega$. 
Proof We follow [29, Theorem 4.1]. Also, see [15, Lemma 3.3]. Let $k \geq 1$. By the change of variable $v(x):=|x|^{\beta} u(x)$ we can check that $v$ solves

$$
\begin{cases}L_{\beta} v=\frac{\mu}{\left.|x|^{\beta(1-\gamma)}\right)_{\nu}}+|x|^{-\beta} f & \text { in } \Omega, \\ \nu>0 & \text { in } \Omega, \\ \nu=0 & \text { in }\left(\mathbb{R}^{N} \backslash \Omega\right),\end{cases}
$$

where the operator $L_{\beta}$ is as follows:

$$
L_{\beta} v:=C_{N, s} \mathrm{P} . \mathrm{V} \cdot \int_{\mathbb{R}^{N}} \frac{v(x)-v(y)}{|x-y|^{N+2 s}} \frac{d y}{|x|^{\beta}|y|^{\beta}} .
$$

See [29, Sect. 2] for the properties of this operator and the associated weighted fractional Sobolev space.

Using $G_{k}(v)$ as a test function in (57) and following the proof of [29, Theorem 4.1], we obtain

$$
\begin{aligned}
\frac{C_{N, s}}{2} \iint_{D_{\Omega}} \frac{\left|G_{k}(v(x))-G_{k}(v(y))\right|^{2}}{|x-y|^{N+2 s}} \frac{d x}{|x|^{\beta}} \frac{d y}{|x|^{\beta}} \leq & \int_{A_{k}}|x|^{\beta \gamma} \frac{\mu}{v^{\gamma}} G_{k}(v) \frac{d x}{|x|^{\beta}} \\
& +\int_{A_{k}} f G_{k}(v) \frac{d x}{|x|^{\beta}}
\end{aligned}
$$

where $A_{k}:=\{x \in \Omega: v(x) \geq k\}$. Applying the weighted Sobolev inequality [29, Proposition 2.11] to the left-hand side of (58) and noting that $|x|^{\beta \gamma} \leq C_{2}$ in $\Omega$, we have

$$
C_{1}\left\|G_{k}(v)\right\|_{L^{2^{*}}\left(\Omega,|x|^{-\beta} d x\right)}^{2} \leq C_{2} \int_{A_{k}} \frac{\mu}{v^{\gamma}} G_{k}(v) \frac{d x}{|x|^{\beta}}+\int_{A_{k}} f G_{k}(v) \frac{d x}{|x|^{\beta}} .
$$

For the first term on the right-hand side of this inequality, by using the Hölder inequality we get

$$
\left|\int_{A_{k}} \frac{\mu}{v^{\gamma}} G_{k}(v) \frac{d x}{|x|^{\beta}}\right| \leq k^{-\gamma}\|\mu\|_{L^{m}(\Omega)}\left\|G_{k}(v)\right\|_{L^{2 *}\left(\Omega,|x|^{-\beta} d x\right)}\left|A_{k}\right|^{1-\frac{1}{2_{s}^{*}}-\frac{1}{m}} .
$$

Similarly, for the second term,

$$
\left|\int_{A_{k}} f G_{k}(v) \frac{d x}{|x|^{\beta}}\right| \leq\|f\|_{L^{m}(\Omega)}\left\|G_{k}(v)\right\|_{L^{2 *}\left(\Omega,|x|^{-\beta} d x\right)}\left|A_{k}\right|^{1-\frac{1}{2_{s}^{*}-\frac{1}{m}}} .
$$

Putting these results together, we obtain

$$
\left.\left\|G_{k}(v)\right\|_{L^{2 *}\left(\Omega,|x|^{-\beta}\right.} d x\right) \leq C_{3}\left|A_{k}\right|^{1-\frac{1}{2_{s}^{*}}-\frac{1}{m}} .
$$

On the other hand, since $\Omega$ is bounded, there exists a constant $C_{4}>0$ such that

$$
\left\|G_{k}(v)\right\|_{L^{2_{s}^{*}}\left(\Omega,|x|^{-\beta} d x\right)} \geq C_{4}\left\|G_{k}(v)\right\|_{L^{2_{s}^{*}(\Omega)}} .
$$


Moreover, for any $z>k$, we have that $A_{z} \subset A_{k}$ and $G_{k}(v) \chi_{A_{z}} \geq(z-k)$. Thus from (59) and (60) we have

$$
(z-k)\left|A_{z}\right|^{\frac{1}{2 s_{s}^{*}}} \leq C_{5}\left|A_{k}\right|^{1-\frac{1}{2_{s}^{*}}-\frac{1}{m}}
$$

or, equivalently,

$$
\left|A_{z}\right| \leq C_{6} \frac{\left|A_{k}\right|^{2_{s}^{*}\left(1-\frac{1}{\left.2_{s}^{*}-\frac{1}{m}\right)}\right.}}{(z-k)^{2_{s}^{*}}} .
$$

Now by invoking [29, Lemma 2.23] with $\psi(h):=\left|A_{h}\right|$ and noting that $2_{s}^{*}\left(1-\frac{1}{2_{s}^{*}}-\frac{1}{m}\right)>1$ because of $m>\frac{N}{2 s}$, we obtain that there exists $k_{0}$ such that $\psi(k) \equiv 0$ for any $k \geq k_{0}$. Thus $v(x) \leq k_{0}$ a.e. in $\Omega$. This means that $u(x) \leq k_{0}|x|^{-\beta}$ a.e. in $\Omega$.

\section{The parabolic case and a stabilization result}

In this section, we study on the following evolution problem:

$$
\begin{cases}u_{t}+(-\Delta)^{s} u=\lambda \frac{u}{|x|^{2 s}}+\frac{1}{u^{\gamma}}+f(x, t) & \text { in } \Omega \times(0, T), \\ u>0 & \text { in } \Omega \times(0, T), \\ u=0 & \text { in }\left(\mathbb{R}^{N} \backslash \Omega\right) \times(0, T), \\ u(x, 0)=u_{0} & \text { in } \mathbb{R}^{N},\end{cases}
$$

where $u_{0} \in X_{0}^{s}(\Omega)$ satisfies an appropriate cone condition, which will be specified later. In what follows, we consider the existence and uniqueness and also a stabilization for problem (61).

First of all, we define the notion of a weak solution. We need the following class of test functions:

$$
\mathcal{A}\left(\Omega_{T}\right):=\left\{u: u \in L^{2}(\Omega \times(0, T)), u_{t} \in L^{2}(\Omega \times(0, T)), u \in L^{\infty}\left(0, T ; X_{0}^{s}(\Omega)\right)\right\} .
$$

Note that the Aubin-Lions-Simon lemma (see [56]) implies that the following embedding is compact:

$$
\mathcal{A}\left(\Omega_{T}\right) \hookrightarrow C\left([0, T] ; L^{2}(\Omega)\right) .
$$

Definition 5.1 Let $u_{0} \in L^{2}(\Omega)$ and $f \in L^{2}(\Omega \times(0, T))$. We say that $u \in \mathcal{A}\left(\Omega_{T}\right)$ is a weak supersolution (subsolution) to problem (61) if

- for every $K \Subset \Omega \times(0, T)$, there exists $C_{K}>0$ such that $u(x, t) \geq C_{K}$ a.e. in $K$ and also $u \equiv 0$ in $\left(\mathbb{R}^{N} \backslash \Omega\right) \times[0, T)$;

- for every nonnegative $\phi \in \mathcal{A}\left(\Omega_{T}\right)$, we have

$$
\begin{aligned}
& \int_{0}^{T} \int_{\Omega} u_{t} \phi d x d t+\int_{0}^{T} \int_{\mathbb{R}^{N}}(-\Delta)^{\frac{s}{2}} u(-\Delta)^{\frac{s}{2}} \phi d x d t \\
& \quad \geq(\leq) \lambda \int_{0}^{T} \int_{\Omega} \frac{u \phi}{|x|^{2 s}} d x d t+\int_{0}^{T} \int_{\Omega} \frac{\phi}{u^{\gamma}} d x d t+\int_{0}^{T} \int_{\Omega} f \phi d x d t
\end{aligned}
$$


and also that the second term on the right-hand side of the inequality is finite for any $\phi \in \mathcal{A}\left(\Omega_{T}\right)$ (the well-posedness of the second term on the right-hand side will be clear after the construction of a solution);

- $u(x, 0) \geq(\leq) u_{0}(x)$ a.e. in $\Omega$.

If $u$ is a weak supersolution and subsolution, then we say that $u$ is a weak solution. Note that by embedding (62) the initial condition $u(x, 0)=u_{0}$ makes sense.

Before outlining our theorems, we need to define the following sets:

- $\mathcal{U}_{\gamma}^{\text {Sing }}$, the set of all functions in $L^{2}(\Omega)$ such that there exists $k_{1}>0$ such that

$$
\begin{cases}k_{1} \delta^{s}(x) \leq|x|^{\beta} u(x), & 0<\gamma<1, \\ k_{1} \delta^{s}(x)\left(\ln \left(\frac{r}{\delta^{s}(x)}\right)\right)^{\frac{1}{2}} \leq|x|^{\beta} u(x), & \gamma=1, \\ k_{1} \delta^{\frac{2 s}{\gamma+1}}(x) \leq|x|^{\beta} u(x), & \gamma>1,\end{cases}
$$

where $r>\operatorname{diam}(\Omega)$;

- $W(\Omega):=\left\{\phi \in C(\bar{\Omega} \backslash\{0\}):|x|^{\beta} \phi \in C(\bar{\Omega})\right\}$ equipped with the $L^{\infty}\left(\Omega,|x|^{\beta} d x\right)$ norm

$$
\|u\|_{L^{\infty}\left(\Omega,|x|^{\beta} d x\right)}:=\operatorname{ess} \sup \left\{|x|^{\beta}|u(x)|: x \in \Omega\right\} .
$$

Also, we need the following definition.

Definition 5.2 We say that $u(t) \in \mathcal{U}_{\gamma}^{\text {Sing }}$ uniformly for $t \in[0, T]$ if there exist $\psi_{1}, \psi_{2} \in \mathcal{U}_{\gamma}^{\text {Sing }}$ such that $\psi_{1}(x) \leq u(x, t) \leq \psi_{2}(x)$ for a.e. $(x, t) \in \Omega \times[0, T]$.

Theorem 5.3 Let $0 \leq g \in L^{\infty}\left(\Omega,|x|^{\beta} d x\right), 0<\lambda<\Lambda_{N, s}$, and $\theta>0$. Then the following problem has a unique weak energy solution $u_{\theta} \in X_{0}^{s}(\Omega) \cap \mathcal{U}_{\gamma}^{\text {Sing }}$ for any $0<\gamma \leq 1$ or $\gamma>1$ with $2 s(\gamma-1)<\gamma+1$ :

$$
\begin{cases}u+\theta\left((-\Delta)^{s} u-\lambda \frac{u}{|x|^{2 s}}-\frac{1}{u^{\gamma}}\right)=g & \text { in } \Omega, \\ u>0 & \text { in } \Omega, \\ u=0 & \text { in }\left(\mathbb{R}^{N} \backslash \Omega\right) .\end{cases}
$$

Moreover, there exists a positive constant $\lambda_{*}<\Lambda_{N, s}$ such that for any $\lambda \in\left(0, \lambda_{*}\right)$, this unique solution also belongs to $W(\Omega)$.

Proof We follow the proof of [30, Theorem 2.4]. For any $\epsilon>0$, consider the following approximating problem:

$$
\begin{cases}u_{\epsilon, \theta}+\theta\left((-\Delta)^{s} u_{\epsilon, \theta}-\lambda \frac{u_{\epsilon, \theta}}{|x|^{2 s}}-\frac{1}{\left(u_{\epsilon, \theta}+\epsilon\right)^{\gamma}}\right)=g & \text { in } \Omega, \\ u_{\epsilon, \theta}>0 & \text { in } \Omega, \\ u_{\epsilon, \theta}=0 & \text { in }\left(\mathbb{R}^{N} \backslash \Omega\right) .\end{cases}
$$

The existence of a unique energy solution easily follows by the classical variational methods. Indeed, let $X_{0}^{s}(\Omega)^{+}:=\left\{u \in X_{0}^{s}(\Omega) \mid u \geq 0\right\}$, and consider the corresponding energy func- 
tional to problem (64):

$$
\begin{aligned}
I_{\epsilon, \theta}(u)= & \frac{1}{2} \int_{\Omega} u^{2} d x+\frac{\theta C_{N, s}}{4}\|u\|_{X_{0}^{s}(\Omega)}^{2}-\frac{\theta \lambda}{2} \int_{\Omega} \frac{u^{2}}{|x|^{2 s}} d x \\
& -\frac{\theta}{1-\gamma} \int_{\Omega}(u+\epsilon)^{1-\gamma} d x-\int_{\Omega} g u d x, \quad u \in X_{0}^{s}(\Omega)^{+} .
\end{aligned}
$$

Note that the last term is well-defined since $g \in L^{\infty}\left(\Omega,|x|^{\beta} d x\right) \subset L^{2}(\Omega)$. Using the Hardy inequality, we can show that this functional $I_{\epsilon, \theta}: X_{0}^{s}(\Omega)^{+} \rightarrow \mathbb{R}$ is weakly lower semicontinuous, coercive, and strictly convex. Since $X_{0}^{s}(\Omega)^{+}$is a closed subspace of the reflexive space $X_{0}^{s}(\Omega)^{+}$, the existence of a unique minimizer is obvious by the classical theory (e.g., see [57, Chap. 1]). Therefore, as a consequence, we get the existence of a unique energy solution to problem (64).

Let $0<\epsilon_{1} \leq \epsilon_{2}$. We want to show that $u_{\epsilon_{2}, \theta} \leq u_{\epsilon_{1}, \theta}$ a.e. in $\Omega$. This easily follows by subtracting the weak formulations of $u_{\epsilon_{i}, \theta}, i=1,2$, and using $\left(u_{\epsilon_{2}, \theta}-u_{\epsilon_{1}, \theta}\right)^{+}$as a test function, which, together with the Hardy inequality, implies $\left(u_{\epsilon_{2}, \theta}-u_{\epsilon_{1}, \theta}\right)^{+} \equiv 0$ a.e. in $\Omega$. Now let $w \in X_{0}^{s}(\Omega) \cap \mathcal{U}_{\gamma}^{\text {Sing }}$ be the unique energy solution to

$$
\begin{cases}(-\Delta)^{s} w=\lambda \frac{w}{|x|^{2 s}}+w^{-\gamma} & \text { in } \Omega, \\ w>0 & \text { in } \Omega, \\ w=0 & \text { in }\left(\mathbb{R}^{N} \backslash \Omega\right) .\end{cases}
$$

Note that for general $\gamma>1$, we only know that $w \in X_{\mathrm{loc}}^{s}(\Omega)$. However, since $2 s(\gamma-1)<\gamma+1$, thanks to Remark 6, we also get $w \in X_{0}^{s}(\Omega)$.

Now define $\bar{u}:=M w$ for some $M>1$. Because of the same singular behavior of $w$ and $g$ near the origin, noting that $g$ is bounded near the boundary $\partial \Omega$ and $w$ behaves as $c \delta^{s}$ near the boundary, we can choose $M$ large enough (independent of $\epsilon$ ) such that

$$
\begin{aligned}
\bar{u}+\theta\left((-\Delta)^{s} \bar{u}-\lambda \frac{\bar{u}}{|x|^{2 s}}-\frac{1}{(\bar{u}+\epsilon)^{\gamma}}\right) & =M w+\theta\left(\frac{M}{w^{\gamma}}-\frac{1}{(M w+\epsilon)^{\gamma}}\right) \\
& \geq M w+\theta\left(\frac{1}{(M w)^{\gamma}}-\frac{1}{(M w+\epsilon)^{\gamma}}\right) \\
& >\operatorname{gin} \Omega .
\end{aligned}
$$

Since $A_{\theta}: X_{0}^{s}(\Omega) \cap \mathcal{U}_{\gamma}^{\text {Sing }} \rightarrow X^{-s}(\Omega), A_{\theta}(u):=u+\theta\left((-\Delta)^{s} u-\lambda \frac{u}{|x|^{2 s}}-u^{-\gamma}\right)$ is a strictly monotone operator for $0<\lambda<\Lambda_{N, s}$ (this strict monotonicity is an easy consequence of [30, Lemma 3.1] and the Hardy inequality), and thus $u_{\epsilon, \theta} \leq \bar{u}$. Therefore $u_{\theta} \leq \bar{u}$, where $u_{\theta}:=\lim _{\epsilon \rightarrow 0^{+}} u_{\epsilon, \theta}$. This implies that $u_{\theta}$ is a very weak (distributional) solution to problem (63), that is,

$$
\int_{\Omega} u_{\theta} \phi d x+\theta\left(\int_{\mathbb{R}^{N}} u_{\theta}(-\Delta)^{s} \phi d x-\lambda \int_{\Omega} \frac{u_{\theta}}{|x|^{2 s}} \phi d x-\int_{\Omega} \frac{\phi}{u_{\theta}^{\gamma}} d x\right)=\int_{\Omega} g \phi d x
$$

for any $\phi \in \mathcal{T}(\Omega)$. In fact, we want to show that $u_{\theta}$ is an energy solution. For this purpose, let $\underline{u}:=m w$ for some $m>0$. If we choose $m$ small enough such that

$$
m^{\gamma+1}\left(1+\frac{w^{\gamma+1}}{\theta}\right) \leq 1+m^{\gamma} \frac{g w^{\gamma}}{\theta} \quad \text { in } \Omega
$$


(which is possible by taking into consideration the behavior of $w$ and $g$ near the origin and the boundary $\partial \Omega$ ), then $\underline{u}$ will be a subsolution to problem (63), and by similar arguments as before we obtain $\underline{u} \leq u_{\theta}$ a.e. in $\Omega$. Thus $\underline{u} \leq u_{\theta} \leq \bar{u}$, which implies that $u_{\theta} \in \mathcal{U}_{\gamma}^{\text {Sing }}$. On the other hand, by invoking the Hardy inequality, because of the restrictions $0<\gamma \leq 1$ or $\gamma>1$ with $2 s(\gamma-1)<\gamma+1$, a density argument shows that (65) holds for all $\phi \in X_{0}^{s}(\Omega)$ (see Remark 6). This means that $u_{\theta} \in X_{0}^{s}(\Omega)$ is the unique energy solution to problem (63). Now let $g \in L^{m}(\Omega), m>\frac{N}{2 s}$, which is possible if $m \beta<N$ or, equivalently, $\alpha>\frac{N-2 s}{2}-\frac{N}{m}$. Since $\lambda=\lambda(\alpha)$ given by (9) is a continuous decreasing function for $\alpha \in\left[0, \frac{N-2 s}{2}\right)$, this recent condition is equivalent to $0<\lambda<\lambda_{*}$ for some $\lambda_{*}<\Lambda_{N, s}$. Thus the comparison principle for the fractional Laplacian operator, together with Theorem 4.3, gives $u(x) \leq C|x|^{-\beta}$ a.e. in $\mathbb{R}^{N}$. Now the interior regularity theory for the fractional Laplacian, which follows from [37, Proposition 1.1], implies that $u \in C\left(\tilde{\Omega} \backslash B_{\epsilon}(0)\right)$ for any $\tilde{\Omega} \Subset \Omega$ and any $\epsilon>0$ small enough. Moreover, by following the proof of [49, Theorem 1.4] we obtain the continuity of $u$ up to the boundary of $\Omega$. This completes the proof.

Thanks to the Hardy inequality, following the idea of [30, Theorem 4.1], that is. applying the semidiscretization in time with implicit Euler method and also invoking the result of Theorem 5.3, we obtain the following existence result to problem (61).

Theorem 5.4 Let $s \in(0,1)$, let either $0<\gamma \leq 1$ or $\gamma>1$ with $2 s(\gamma-1)<\gamma+1$, and let $0<\lambda<\Lambda_{N, s}$. Also, assume that $u_{0} \in X_{0}^{s}(\Omega) \cap \mathcal{U}_{\gamma}^{\text {Sing }}$ and $0 \leq f(x, t) \leq|x|^{\gamma \beta}, 0 \leq t \leq T$. Then there is a unique positive weak solution in $\mathcal{A}\left(\Omega_{T}\right) \cap \mathcal{U}_{\gamma}^{\text {Sing }}$ to problem (61). Moreover, $u$ belongs to $C\left([0, T], X_{0}^{s}(\Omega)\right), u(t) \in \mathcal{U}_{\gamma}^{\text {Sing }}$ uniformly for $t \in[0, T]$, and for any $t \in[0, T]$,

$$
\begin{aligned}
\int_{0}^{t} \int_{\Omega}\left|\frac{\partial u}{\partial \tau}\right|^{2} d x d \tau+\frac{C_{N, s}}{2}\|u(x, t)\|_{X_{0}^{s}(\Omega)}^{2}-\lambda \int_{\Omega} \frac{u^{2}(x, t)}{|x|^{2 s}} d x \\
\quad-\frac{1}{1-\gamma} \int_{\Omega} u^{1-\gamma}(x, t) d x \\
=\int_{0}^{t} \int_{\Omega} f(x, t) \frac{\partial u}{\partial \tau} d x d \tau+\frac{C_{N, s}}{2}\left\|u_{0}(x)\right\|_{X_{0}^{s}(\Omega)}^{2}-\lambda \int_{\Omega} \frac{u_{0}^{2}}{|x|^{2 s}} d x \\
\quad-\frac{1}{1-\gamma} \int_{\Omega} u_{0}^{1-\gamma}(x) d x .
\end{aligned}
$$

In addition, if $0<\lambda<\lambda_{*}\left(\lambda_{*}\right.$ is as in Theorem 5.3) and $\left.u_{0} \in \overline{\mathcal{D}}(L)^{L^{\infty}\left(\Omega,|x|^{\beta}\right.} d x\right)$, where

$$
\mathcal{D}(L):=\left\{v \in X_{0}^{s}(\Omega) \cap \mathcal{U}_{\gamma}^{\text {Sing }} \cap W(\Omega) \mid L(v):=(-\Delta)^{s} v-\lambda \frac{v}{|x|^{2 s}}-\frac{1}{v^{\gamma}} \in L^{\infty}\left(\Omega,|x|^{\beta} d x\right)\right\},
$$

then the solution obtained above belongs to $C([0, T] ; W(\Omega))$.

Remark 8 By invoking [42, Proposition 5.3] it is straightforward to obtain that if $\lambda>\Lambda_{N, s}$, then problem (61) has no solution. Moreover, a similar complete blowup phenomenon occurs as in the stationary case.

Finally, the following theorem is on stabilization to problem (61). By stabilization we mean that if $\hat{u}(x)$ is the unique solution to a stationary problem with the datum of $f(x)$, then $u(x, t)$, the solution to the parabolic problem, converges to $\hat{u}(x)$ as $t \rightarrow \infty$. 
Theorem 5.5 Let $s \in(0,1)$, let either $0<\gamma \leq 1$ or $\gamma>1$ with $2 s(\gamma-1)<\gamma+1$, and let $0<\lambda<\lambda_{*}$. Also assume that $\left.u_{0} \in \overline{\mathcal{D}}(L)^{L^{\infty}\left(\Omega,|x|^{\beta}\right.} d x\right)$ and $0 \leq f(x, t)=f(x) \leq|x|^{\gamma \beta}, 0 \leq t \leq T$. Then if $u(x, t)$ is the unique positive weak solution to problem (61), then

$$
u(x, t) \rightarrow \hat{u}(x) \quad \operatorname{in} L^{\infty}\left(\Omega,|x|^{\beta} d x\right) \text { as } t \rightarrow+\infty
$$

where $\hat{u}$ is the unique weak solution to (1) with $\mu \equiv 1$.

Since proofs of the theorems in this section are essentially the same as those of the corresponding ones in [30], we give them in the Appendix.

\section{Appendix}

Here we give the proofs of Theorems 5.4 and 5.5 .

Proof of Theorem 5.4 We follow the proofs of [30, Theorem 4.1, Theorem 4.2, and Proposition 2.8].

Let $\eta_{t}=\frac{T}{n}$ and for $0 \leq k \leq n$, define $t_{k}=k \eta_{t}$ and

$$
f_{k}(x):=\frac{1}{\eta_{t}} \int_{t_{k-1}}^{t_{k}} f(x, \tau) d \tau, \quad \forall x \in \Omega
$$

Also, define

$$
f_{\eta_{t}}(x, t):= \begin{cases}f_{1}(x), & 0 \leq t<t_{1}, \\ f_{2}(x), & t_{1} \leq t<t_{2}, \\ \vdots & \vdots \\ f_{n}(x), & t_{n-1} \leq t<t_{n} .\end{cases}
$$

Clearly, we have $f_{\eta_{t}}(\cdot, t) \in L^{\infty}\left(\Omega,|x|^{\beta} d x\right) \subset L^{2}(\Omega), t \in[0, T]$, and for $1<p<+\infty$,

$$
\left\|f_{\eta_{t}}\right\|_{L^{p}(\Omega \times(0, T))} \leq(|\Omega| T)^{\frac{1}{p}}\|f\|_{L^{p}(\Omega \times(0, T))}
$$

Now let $\theta=\eta_{t}$, and let $g=\eta_{t} f_{k}+u_{k-1} \in L^{\infty}\left(\Omega,|x|^{\beta} d x\right)$ in problem (63). Then Theorem 5.3 implies the existence of a solution $u_{k} \in X_{0}^{S}(\Omega) \cap \mathcal{U}_{\gamma}^{\text {Sing }}$ to the following problem:

$$
\begin{cases}\frac{u_{k}-u_{k-1}}{\eta_{t}}+\left((-\Delta)^{s} u_{k}-\lambda \frac{u_{k}}{|x|^{2 s}}-\frac{1}{u_{k}^{\gamma}}\right)=f_{k} & \text { in } \Omega, \\ u_{k}>0 & \text { in } \Omega, \\ u_{k}=0 & \text { in }\left(\mathbb{R}^{N} \backslash \Omega\right),\end{cases}
$$

where the above iteration starts from the initial condition $u_{0}(x)$ of problem (61).

Now, for $1 \leq k \leq n$ and $t \in\left[t_{k-1}, t_{k}\right)$, inspired by the implicit Euler method, we define

$$
\left\{\begin{array}{l}
u_{\eta_{t}}(x, t):=u_{k}(x), \\
\tilde{u}_{\eta_{t}}(x, t):=\frac{u_{k}(x)-u_{k-1}(x)}{\eta_{t}}\left(t-t_{k-1}\right)+u_{k-1}(x) .
\end{array}\right.
$$


The functions $u_{\eta_{t}}$ and $\tilde{u}_{\eta_{t}}$ satisfy

$$
\frac{\partial \tilde{u}_{\eta_{t}}}{\partial t}+\left((-\Delta)^{s} u_{\eta_{t}}-\lambda \frac{u_{\eta_{t}}}{|x|^{2 s}}-\frac{1}{u_{\eta_{t}}^{\gamma}}\right)=f_{\eta_{t}} .
$$

Now in what follows, we establish some uniform estimates in $\eta_{t}$ for $u_{\eta_{t}}$ and $\tilde{u}_{\eta_{t}}$.

Multiplying (68) by $\eta_{t} u_{k}$, integrating over $\mathbb{R}^{N}$, summing from $k=1$ to $n^{\prime} \leq n$, and using Young's inequality, (67), and embedding (6), we get, for a constant $C>0$,

$$
\begin{aligned}
& \sum_{k=1}^{n^{\prime}} \int_{\Omega}\left(u_{k}-u_{k-1}\right) u_{k} d x+\eta_{t} \sum_{k=1}^{n^{\prime}}\left(\frac{C_{N, s}}{2}\left\|u_{k}\right\|_{X_{0}^{s}(\Omega)}^{2}-\lambda \int_{\Omega} \frac{\left(u_{k}\right)^{2}}{|x|^{2 s}} d x-\int_{\Omega} \frac{1}{u_{k}^{\gamma-1}} d x\right) \\
& \quad=\eta_{t} \sum_{k=1}^{n^{\prime}} \int_{\Omega} f_{k} u_{k} d x \\
& \quad \leq \eta_{t} \sum_{k=1}^{n^{\prime}} \int_{\Omega} \frac{\left|f_{k}\right|^{2}}{2} d x+\eta_{t} \sum_{k=1}^{n^{\prime}} \int_{\Omega} \frac{\left|u_{k}\right|^{2}}{2} d x \\
& \quad \leq \frac{T|\Omega|}{2}\|f\|_{L^{\infty}(\Omega \times(0, T))}^{2}+\frac{C \eta_{t}}{2} \sum_{k=1}^{n^{\prime}}\left\|u_{k}\right\|_{X_{0}^{s}(\Omega)}^{2} .
\end{aligned}
$$

For the first term in the left-hand side of (70), similarly to (2.7) in the proof of [31, Theorem 0.9 ], we have the equality

$$
\begin{aligned}
\sum_{k=1}^{n^{\prime}} \int_{\Omega}\left(u_{k}-u_{k-1}\right) u_{k} d x= & \frac{1}{2} \sum_{k=1}^{n^{\prime}} \int_{\Omega}\left|u_{k}-u_{k-1}\right|^{2} d x \\
& +\frac{1}{2} \int_{\Omega}\left|u_{n^{\prime}}\right|^{2} d x-\frac{1}{2} \int_{\Omega}\left|u_{0}\right|^{2} d x .
\end{aligned}
$$

Now, let $w \in X_{0}^{s}(\Omega) \cap \mathcal{U}_{\gamma}^{\text {Sing }}$ solve

$$
\begin{cases}(-\Delta)^{s} w=\lambda \frac{w}{|x|^{2 s}}+\frac{1}{w^{\gamma}} & \text { in } \Omega, \\ w>0 & \text { in } \Omega, \\ w=0 & \text { in }\left(\mathbb{R}^{N} \backslash \Omega\right),\end{cases}
$$

and define $\underline{u}=m w, m>0$, and $\bar{u}=M w, M>0$. By a direct computation we have

$$
(-\Delta)^{s} \underline{u}-\lambda \frac{\underline{u}}{|x|^{2 s}}-\frac{1}{\underline{u}^{\gamma}}=\frac{m^{\gamma+1}-1}{m^{\gamma} w^{\gamma}}
$$

and

$$
(-\Delta)^{s} \bar{u}-\lambda \frac{\bar{u}}{|x|^{2 s}}-\frac{1}{\bar{u}^{\gamma}}=\frac{M^{\gamma+1}-1}{M^{\gamma} w^{\gamma}} .
$$


Since $w$ behaves as $c_{1}|x|^{-\beta}$ near the origin and behaves as $c_{2} \delta^{s}$ near the boundary $\partial \Omega$, we can choose $m>0$ small enough and $M>0$ large enough such that

$$
\begin{cases}(-\Delta)^{s} \underline{u}-\lambda \frac{\underline{u}}{|x|^{2 s}}-\frac{1}{\underline{u}^{\gamma}} \leq-|x|^{\gamma \beta} & \text { in } \Omega \\ \underline{u}=0 & \text { in }\left(\mathbb{R}^{N} \backslash \Omega\right)\end{cases}
$$

and

$$
\begin{cases}(-\Delta)^{s} \bar{u}-\lambda \frac{\bar{u}}{|x|^{2 s}}-\frac{1}{\bar{u}^{\gamma}} \geq|x|^{\gamma \beta} & \text { in } \Omega \\ \bar{u}=0 & \text { in }\left(\mathbb{R}^{N} \backslash \Omega\right)\end{cases}
$$

Since $u_{0} \in \mathcal{U}_{\gamma}^{\text {Sing }}$, we can choose $\underline{u}$ and $\bar{u}$ such that it satisfies the above inequalities and $\underline{u} \leq u_{0} \leq \bar{u}$. By the monotonicity of the operator $(-\Delta)^{s} u-\lambda \frac{u}{|x|^{2 s}}-u^{-\gamma}$, applying it iteratively, we get $\underline{u} \leq u_{k} \leq \bar{u}$ for all $k$. This implies for a.e. $(x, t) \in[0, T] \times \Omega$,

$$
\underline{u}(x) \leq u_{\eta_{t}}, \quad \tilde{u}_{\eta_{t}}(x, t) \leq \bar{u}(x) .
$$

Thus $u_{\eta_{t}}, \tilde{u}_{\eta_{t}} \in \mathcal{U}_{\gamma}^{\text {Sing }}$ uniformly for $t \in[0, T]$. Now we can estimate the singular term in (70) as follows:

$$
\eta_{t} \sum_{n=1}^{n^{\prime}} \int_{\Omega} \frac{1}{u_{k}^{\gamma}} d x \leq \begin{cases}T \int_{\Omega} \bar{u}^{1-\gamma} d x<+\infty, & 0<\gamma \leq 1, \\ T \int_{\Omega} \underline{u}^{1-\gamma} d x<+\infty, & \gamma>1 \text { with } 2 s(\gamma-1)<\gamma+1 .\end{cases}
$$

By the definition of $u_{\eta_{t}}$ and $\tilde{u}_{\eta_{t}}$, noting that $u_{k} \in L^{\infty}\left(\Omega,|x|^{\beta} d x\right)$ for all $k$, we obtain that

$$
u_{\eta_{t}}, \tilde{u}_{\eta_{t}} \text { are bounded in } L^{\infty}\left([0, T] ; L^{\infty}\left(\Omega,|x|^{\beta} d x\right)\right)
$$

On the other hand, for $t \in\left[t_{k-1}, t_{k}\right)$, we have

$$
\begin{aligned}
\left\|\tilde{u}_{\eta t}(t, \cdot)\right\|_{X_{0}^{s}(\Omega)} & =\left\|\frac{\left(t-t_{k-1}\right)}{\eta_{t}} u_{k}+\frac{\eta_{t}-t+t_{k-1}}{\eta_{t}} u_{k-1}\right\|_{X_{0}^{s}(\Omega)} \\
& \leq\left\|u_{k}\right\|_{X_{0}^{s}(\Omega)}+\left\|u_{k-1}\right\|_{X_{0}^{s}(\Omega)} .
\end{aligned}
$$

Integrating both sides of (70) over $\left(t_{k-1}, t_{k}\right)$ and using the above estimates, the Hardy Inequality, and (71), we get that

$$
u_{\eta_{t}}, \tilde{u}_{\eta_{t}} \text { are bounded in } L^{2}\left([0, T] ; X_{0}^{s}(\Omega)\right) \text {. }
$$

Now we want to obtain another a priori estimate. 
Multiplying (68) by $u_{k}-u_{k-1}$, integrating over $\mathbb{R}^{N}$, summing from $k=1$ to $n^{\prime} \leq n$, and using Young's inequality, we get

$$
\begin{aligned}
& \eta_{t} \sum_{k=1}^{n^{\prime}} \int_{\Omega}\left(\frac{u_{k}-u_{k-1}}{\eta_{t}}\right)^{2} d x+\sum_{k=1}^{n^{\prime}} \int_{\mathbb{R}^{N}}\left((-\Delta)^{s} u_{k}(x)\right)\left(u_{k}-u_{k-1}\right)(x) d x \\
& \quad-\lambda \sum_{k=1}^{n^{\prime}} \int_{\Omega} \frac{u_{k}\left(u_{k}-u_{k-1}\right)}{|x|^{2 s}} d x-\sum_{k=1}^{n^{\prime}} \int_{\Omega} \frac{u_{k}-u_{k-1}}{u_{k}^{\gamma}} d x \\
& =\eta_{t} \sum_{k=1}^{n^{\prime}} \int_{\Omega} \frac{f_{k}\left(u_{k}-u_{k-1}\right)}{\eta_{t}} d x \\
& \leq \frac{\eta_{t}}{2} \sum_{k=1}^{n^{\prime}}\left(\int_{\Omega}\left|f_{k}\right|^{2} d x+\int_{\Omega}\left(\frac{u_{k}-u_{k-1}}{\eta_{t}}\right)^{2} d x\right)
\end{aligned}
$$

which implies

$$
\begin{aligned}
& \frac{\eta_{t}}{2} \sum_{k=1}^{n^{\prime}} \int_{\Omega}\left(\frac{u_{k}-u_{k-1}}{\eta_{t}}\right)^{2} d x+\sum_{k=1}^{n^{\prime}} \int_{\mathbb{R}^{N}}\left((-\Delta)^{s} u_{k}(x)\right)\left(u_{k}-u_{k-1}\right)(x) d x \\
& \quad-\lambda \sum_{k=1}^{n^{\prime}} \int_{\Omega} \frac{u_{k}\left(u_{k}-u_{k-1}\right)}{|x|^{2 s}} d x-\sum_{k=1}^{n^{\prime}} \int_{\Omega} \frac{u_{k}-u_{k-1}}{u_{k}^{\gamma}} d x \\
& \quad \leq \frac{|\Omega| T}{2} \sup _{0 \leq t \leq T}\|f(\cdot, t)\|_{L^{2}(\Omega)^{\circ}}^{2}
\end{aligned}
$$

By using the convexity of the term $-\frac{1}{1-\gamma} \int_{\Omega} u^{1-\gamma} d x$ we get

$$
\frac{1}{1-\gamma} \int_{\Omega}\left(u_{k-1}^{1-\gamma}-u_{k}^{1-\gamma}\right) d x \leq-\int_{\Omega} \frac{u_{k}-u_{k-1}}{u_{k}^{\gamma}} d x
$$

Also, we have

$$
\frac{C_{N, s}}{2}\left(\left\|u_{k}\right\|_{X_{0}^{s}(\Omega)}^{2}-\left\|u_{k-1}\right\|_{X_{0}^{s}(\Omega)}^{2}\right) \leq \int_{\mathbb{R}^{N}}\left((-\Delta)^{s} u_{k}(x)\right)\left(u_{k}-u_{k-1}\right)(x) d x
$$

and

$$
\int_{\Omega} \frac{\left(u_{k}\right)^{2}-\left(u_{k-1}\right)^{2}}{|x|^{2 s}} d x \leq \int_{\Omega} \frac{u_{k}\left(u_{k}-u_{k-1}\right)}{|x|^{2 s}} d x
$$

Therefore (76), together with (77), (78), and (79), gives

$$
\begin{aligned}
& \frac{\eta_{t}}{2} \sum_{k=1}^{n^{\prime}} \int_{\Omega}\left(\frac{u_{k}-u_{k-1}}{\eta_{t}}\right)^{2} d x+\frac{C_{N, s}}{2}\left(\left\|u_{n^{\prime}}\right\|_{X_{0}^{s}(\Omega)}^{2}-\left\|u_{0}\right\|_{X_{0}^{s}(\Omega)}^{2}\right) \\
& \quad-\lambda \int_{\Omega} \frac{\left(u_{n^{\prime}}\right)^{2}-\left(u_{0}\right)^{2}}{|x|^{2 s}} d x+\frac{1}{1-\gamma} \int_{\Omega}\left(\left(u_{0}\right)^{1-\gamma}-\left(u_{n^{\prime}}\right)^{1-\gamma}\right) d x \\
& \quad \leq \frac{|\Omega| T}{2} \sup _{0 \leq t \leq T}\|f(\cdot, t)\|_{L^{2}(\Omega)^{\prime}}^{2}
\end{aligned}
$$


Integrating both sides of (80) over $\left(t_{k-1}, t_{k}\right)$ and using (73) and the Hardy inequality, we get

$$
\frac{\eta_{t}}{2} \int_{0}^{T} \int_{\Omega}\left|\frac{\partial \tilde{u}_{\eta_{t}}}{\partial t}\right|^{2} d x d t<+\infty
$$

which implies

$$
\frac{\partial \tilde{u}_{\eta_{t}}}{\partial t} \text { is bounded in } L^{2}(\Omega \times(0, T)) \text { uniformly in } \eta_{t} .
$$

Also, using the definition of $u_{\eta_{t}}$ and $\tilde{u}_{\eta_{t}}$, we obtain that

$$
u_{\eta_{t}} \text { and } \tilde{u}_{\eta_{t}} \text { are bounded in } L^{\infty}\left([0, T] ; X_{0}^{s}(\Omega)\right) \text { uniformly in } \eta_{t} \text {. }
$$

Moreover, there exists a constant $C>0$ (independent of $\eta_{t}$ ) such that

$$
\left\|u_{\eta_{t}}-\tilde{u}_{\eta_{t}}\right\|_{L^{\infty}\left([0, T] ; L^{2}(\Omega)\right)} \leq \max _{1 \leq k \leq n}\left\|u_{k}-u_{k-1}\right\|_{L^{2}(\Omega)} \leq C\left(\eta_{t}\right)^{\frac{1}{2}} .
$$

Now (74) and (82) imply that

$u_{\eta_{t}}$ and $\tilde{u}_{\eta_{t}}$ are bounded in $L^{\infty}\left([0, T] ; X_{0}^{s}(\Omega) \cap L^{\infty}\left(\Omega,|x|^{\beta} d x\right)\right)$ uniformly in $\eta_{t}$.

Therefore, up to a subsequence, as $\eta_{t} \rightarrow 0^{+}$(i.e., $n \rightarrow \infty$ ),

$$
\begin{aligned}
& \tilde{u}_{\eta_{t}} \rightarrow u \text { and } u_{\eta_{t}} \rightarrow v \text { star-weakly in } L^{\infty}\left([0, T] ; X_{0}^{s}(\Omega) \cap L^{\infty}\left(\Omega,|x|^{\beta} d x\right)\right), \\
& \frac{\partial \tilde{u}_{\eta_{t}}}{\partial t} \rightarrow \frac{\partial u}{\partial t} \text { weakly in } L^{2}(\Omega \times(0, T)),
\end{aligned}
$$

where $u, v \in L^{\infty}\left([0, T] ; X_{0}^{s}(\Omega) \cap L^{\infty}\left(\Omega,|x|^{\beta} d x\right)\right)$, and $\frac{\partial u}{\partial t} \in L^{2}(\Omega \times(0, T))$. From (83) we deduce that $u \equiv v$. Also, from (72) we get that $\underline{u} \leq u \leq \bar{u}$. Thus $u \in \mathcal{A}\left(\Omega_{T}\right) \cap \mathcal{U}_{\gamma}^{\text {Sing }}$.

Now we want to show that $u$ is a candidate to the weak solution to (61). By the definition of $\tilde{u}_{\eta_{t}}$ we see that for a.e. $x \in \Omega, \tilde{u}_{\eta_{t}}(\cdot, x) \in C([0, T])$. By (81) we get that $\frac{\partial \tilde{u}_{\eta t}}{\partial t}$ is bounded in $L^{2}(\Omega \times(0, T))$ uniformly in $\eta_{t}$. Also, $\left\{u_{\eta_{t}}\right\}$ is a bounded family in $X_{0}^{s}(\Omega)$. Now let

$$
V:=\left\{u \in C\left([0, T] ; X_{0}^{s}(\Omega)\right): \frac{\partial u}{\partial t} \in L^{2}(\Omega \times(0, T))\right\},
$$

which embeds compactly in $C\left([0, T] ; L^{2}(\Omega)\right)$ by invoking the Aubin-Lions-Simon lemma. Therefore we obtain that $\left\{u_{\eta_{t}}\right\}$ is compactly embedded in the space $C\left([0, T] ; L^{2}(\Omega)\right)$. Now using $\underline{u} \leq \tilde{u}_{\eta_{t}} \leq \bar{u}$, we deduce that $\left\{u_{\eta_{t}}\right\}$ is compactly embedded in $C\left([0, T] ; L^{p}(\Omega)\right), 1<p<$ $\infty$. Thus, up to a subsequence, as $\eta_{t} \rightarrow 0^{+}$,

$$
\tilde{u}_{\eta_{t}} \rightarrow u \quad \text { in } C\left([0, T] ; L^{2}(\Omega)\right) .
$$

Therefore from (85) and (83) we obtain that, as $\eta_{t} \rightarrow 0^{+}$,

$$
u_{\eta_{t}} \rightarrow u \quad \text { in } L^{\infty}\left([0, T] ; L^{2}(\Omega)\right) .
$$


Plugging the test function $\phi=u_{\eta_{t}}-u$ into (69), we obtain

$$
\int_{0}^{T} \int_{\Omega}\left(\frac{\partial \tilde{u}_{\eta_{t}}}{\partial t}+\left((-\Delta)^{s} u_{\eta_{t}}-\lambda \frac{u_{\eta_{t}}}{|x|^{2 s}}-\frac{1}{u_{\eta_{t}}^{\gamma}}\right)\right)\left(u_{\eta_{t}}-u\right) d x d t=\int_{0}^{T} \int_{\Omega} f_{\eta_{t}}\left(u_{\eta_{t}}-u\right) d x d t
$$

Also, since (86) implies that $\int_{0}^{T} \int_{\Omega} \frac{\partial u}{\partial t}\left(\tilde{u}_{\eta_{t}}-u\right) d x d t \rightarrow 0$ as $\eta_{t} \rightarrow 0^{+}$, we get

$$
\begin{aligned}
\int_{0}^{T} & \int_{\Omega}\left(\frac{\partial \tilde{u}_{\eta_{t}}}{\partial t}-\frac{\partial u}{\partial t}\right)\left(\tilde{u}_{\eta_{t}}-u\right) d x d t+\int_{0}^{T}\left\langle(-\Delta)^{s} u_{\eta_{t}}, u_{\eta_{t}}-u\right\rangle d t \\
& -\lambda \int_{0}^{T} \int_{\Omega} \frac{u_{\eta_{t}}\left(u_{\eta_{t}}-u\right)}{|x|^{2 s}} d x d t-\int_{0}^{T} \int_{\Omega} \frac{u_{\eta_{t}}-u}{u_{\eta_{t}}^{\gamma}} d x d t \\
= & \int_{0}^{T} \int_{\Omega} f_{\eta_{t}}\left(u_{\eta_{t}}-u\right) d x d t+\mathrm{o}_{\eta_{t}}(1) .
\end{aligned}
$$

Here $\langle\cdot, \cdot\rangle$ denotes the duality pairing between $X^{-s}(\Omega)$ and $X_{0}^{s}(\Omega)$. By $(72)$ we know that $u_{\eta t}^{\gamma} \leq \underline{u}^{\gamma}$. Also, since $\underline{u} \leq u \leq \bar{u}$, applying the dominated convergence theorem, from (86) we get

$$
\int_{0}^{T} \int_{\Omega} \frac{u_{\eta_{t}}-u}{u_{\eta_{t}}^{\gamma}} d x d t \leq \int_{0}^{T} \int_{\Omega} \frac{u_{\eta_{t}}-u}{\underline{u}^{\gamma}} d x d t=o_{\eta_{t}}(1)
$$

Similarly, using the dominated convergence theorem, from (67) and (86) we obtain

$$
\int_{0}^{T} \int_{\Omega} f_{\eta_{t}}\left(u_{\eta_{t}}-u\right) d x d t=\mathbf{o}_{\eta_{t}}(1)
$$

Now noting that $\tilde{u}_{\eta_{t}}(x, 0)=u(x, 0)=u_{0}$ in a.e. $\Omega$ and applying the integration-by-parts formula, we have

$$
2 \int_{0}^{T} \int_{\Omega}\left(\frac{\partial \tilde{u}_{\eta_{t}}}{\partial t}-\frac{\partial u}{\partial t}\right)\left(\tilde{u}_{\eta_{t}}-u\right) d x d t=\int_{\Omega}\left(\tilde{u}_{\eta_{t}}-u\right)^{2}(T) d t
$$

Therefore, using (87) and the facts that $\int_{0}^{T}\left\langle(-\Delta)^{s} u, u_{\eta_{t}}-u\right\rangle d t=\mathrm{o}_{\eta_{t}}(1)$ and $\int_{0}^{T} \int_{\Omega} \frac{u\left(u_{\eta_{t}}-u\right)}{|x|^{2 s}} d x d t=\mathrm{o}_{\eta_{t}}(1)$, which follow from (86), we obtain

$$
\begin{aligned}
& \frac{1}{2} \int_{\Omega}\left(\tilde{u}_{\eta_{t}}-u\right)^{2}(T) d t+\int_{0}^{T}\left\langle(-\Delta)^{S} u_{\eta_{t}}-(-\Delta)^{s} u, u_{\eta_{t}}-u\right\rangle d t \\
& \quad-\lambda \int_{0}^{T} \int_{\Omega} \frac{\left(u_{\eta_{t}}-u\right)^{2}}{|x|^{2 s}} d x d t=\mathrm{o}_{\eta_{t}}(1) .
\end{aligned}
$$

Now (86), together with the Hardy inequality, gives

$$
\int_{0}^{T}\left\|\left(\tilde{u}_{\eta_{t}}-u\right)(t, \cdot)\right\|_{X_{0}^{s}(\Omega)}^{2} d t=\mathbf{o}_{\eta_{t}}(1)
$$

The above relations imply that, as $\eta_{t} \rightarrow 0^{+}$,

$$
(-\Delta)^{s} u_{\eta_{t}} \rightarrow(-\Delta)^{s} u \quad \text { in } L^{2}\left([0, T] ; X^{-s}(\Omega)\right) .
$$


Using (72) and the fractional Hardy-Sobolev inequality, we obtain the following inequalities for any $\phi \in X_{0}^{s}(\Omega)$ :

$$
\begin{aligned}
& \int_{\Omega}\left|\frac{\phi}{u_{\eta_{t}}^{\gamma}}\right| d x
\end{aligned}
$$

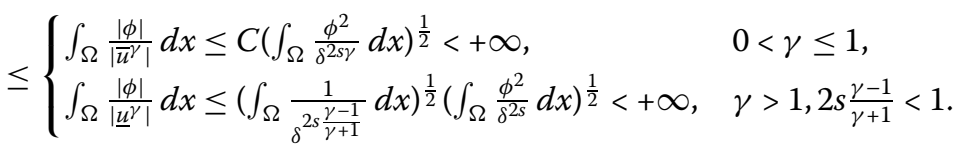

Therefore the dominated convergence theorem implies

$$
\frac{1}{u_{\eta_{t}}^{\gamma}} \rightarrow \frac{1}{u^{\gamma}} \quad \text { in } L^{\infty}\left([0, T] ; X^{-s}(\Omega)\right) \text { as } \eta_{t} \rightarrow 0^{+}
$$

Now we want to show that $u$ satisfies (61) in the weak sense. We already know that

$$
\begin{aligned}
& \int_{0}^{T} \int_{\Omega} \frac{\partial \tilde{u}_{\eta_{t}}}{\partial t} \phi d x d t+\int_{0}^{T} \int_{\mathbb{R}^{N}}(-\Delta)^{s} u_{\eta_{t}} \phi d x d t-\lambda \int_{0}^{T} \int_{\Omega} \frac{u_{\eta_{t}} \phi}{|x|^{2 s}} d x d t \\
& \quad-\int_{0}^{T} \int_{\Omega} \frac{\phi}{u_{\eta_{t}}^{\gamma}} d x d t=\int_{0}^{T} \int_{\Omega} f_{\eta_{t}} \phi d x d t
\end{aligned}
$$

for any $\phi \in \mathcal{A}\left(\Omega_{T}\right)$. Now passing to the limit as $\eta_{t} \rightarrow 0^{+}$and using (67), (84), (88), and (89), we obtain

$$
\begin{aligned}
& \int_{0}^{T} \int_{\Omega} \frac{\partial u}{\partial t} \phi d x d t+\int_{0}^{T} \int_{\mathbb{R}^{N}}(-\Delta)^{s} u \phi d x d t-\lambda \int_{0}^{T} \int_{\Omega} \frac{u \phi}{|x|^{2 s}} d x d t \\
& \quad-\int_{0}^{T} \int_{\Omega} \frac{\phi}{u^{\gamma}} d x d t=\int_{0}^{T} \int_{\Omega} f \phi d x d t .
\end{aligned}
$$

This means that $u$ is the weak solution to (61).

Now we show the uniqueness. Let $u(\cdot, t), v(\cdot, t) \in X_{0}^{s}(\Omega) \cap \mathcal{U}_{\gamma}^{\text {Sing }}$ be weak solutions. Then, for any $t \in[0, T]$, we have

$$
\begin{array}{r}
\int_{\Omega} \frac{\partial(u-v)}{\partial t}(u-v)(x, t) d x+\int_{\mathbb{R}^{N}}\left((-\Delta)^{s}(u-v)\right)(u-v)(x, t) d x \\
-\lambda \int_{\Omega} \frac{(u-v)^{2}(x, t)}{|x|^{2 s}} d x-\int_{\Omega}\left(\frac{1}{u^{\gamma}}-\frac{1}{v^{\gamma}}\right)(u-v)(x, t) d x=0 .
\end{array}
$$

By the Hardy inequality this implies

$$
\begin{aligned}
\frac{\partial}{\partial t}\left(\int_{\Omega} \frac{1}{2}(u-v)^{2}(x, t) d x\right)= & \frac{\Lambda_{N, s}-\lambda}{\Lambda_{N, s}} \cdot \frac{C_{N, s}}{2}\|(u-v)(\cdot, t)\|_{X_{0}^{s}(\Omega)}^{2} \\
& +\int_{\Omega}\left(\frac{1}{u^{\gamma}}-\frac{1}{v^{\gamma}}\right)(u-v)(x, t) d x \leq 0 .
\end{aligned}
$$

Therefore the function $E:[0, T] \rightarrow \mathbb{R}, E(t):=\int_{\Omega} \frac{1}{2}(u-v)^{2}(x, t) d x$, is decreasing. On the other hand, since $u \neq \equiv v$, we get $0<E(t) \leq E(0)=0$, which implies $E(t) \equiv 0$ for all $t \in[0, T]$. This completes the proof of uniqueness. 
Now we prove that $u \in C\left([0, T] ; X_{0}^{s}(\Omega)\right)$. From (85) we already know that $u \in C([0, T]$; $L^{2}(\Omega)$ ), which implies that the map $\tilde{u}:[0, T] \rightarrow X_{0}^{s}(\Omega),[\tilde{u}(t)](x):=u(x, t)$, is weakly continuous. Moreover, from (84) we know that $u \in L^{\infty}\left([0, T] ; X_{0}^{s}(\Omega)\right)$, which implies that $\tilde{u}(t) \in X_{0}^{s}(\Omega)$ and

$$
\|\tilde{u}(t)\|_{X_{0}^{s}(\Omega)} \leq \liminf _{t \rightarrow t_{0}}\|\tilde{u}(t)\|_{X_{0}^{s}(\Omega)}
$$

for all $t_{0} \in[0, T]$.

Now we continue as follows. Multiplying (68) by $u_{k}-u_{k-1}$, integrating over $\mathbb{R}^{N}$, summing from $k=n^{\prime \prime}$ to $n^{\prime}$ ( $n^{\prime}$ was considered in (75)), and using (79), (78), and (77), we get

$$
\begin{aligned}
& \frac{\eta_{t}}{2} \sum_{k=n^{\prime \prime}}^{n^{\prime}} \int_{\Omega}\left(\frac{u_{k}-u_{k-1}}{\eta_{t}}\right)^{2} d x+\frac{C_{N, s}}{2}\left(\left\|u_{n^{\prime}}\right\|_{X_{0}^{s}(\Omega)}^{2}-\left\|u_{n^{\prime \prime}-1}\right\|_{X_{0}^{s}(\Omega)}^{2}\right) \\
& \quad-\lambda \int_{\Omega} \frac{\left(u_{n^{\prime}}\right)^{2}-\left(u_{n^{\prime \prime}}\right)^{2}}{|x|^{2 s}} d x+\frac{1}{1-\gamma} \int_{\Omega}\left(u_{n^{\prime \prime}-1}^{1-\gamma}-u_{n^{\prime}}^{1-\gamma}\right) d x \\
& \leq \sum_{k=n^{\prime \prime}} \int_{\Omega} f_{\eta_{t}}\left(u_{k}-u_{k-1}\right) d x .
\end{aligned}
$$

For any $t_{1} \in\left[t_{0}, T\right]$, we choose $n^{\prime \prime}$ and $n^{\prime}$ in such a way that $n^{\prime \prime} \eta_{t} \rightarrow t_{1}$ and $n^{\prime} \eta_{t} \rightarrow t_{0}$ as $\eta_{t} \rightarrow 0^{+}$. Using (67), (83), (86), and (89) together with (91), we get

$$
\begin{aligned}
\int_{t_{0}}^{t_{1}} & \int_{\Omega}\left(\frac{\partial u}{\partial t}\right)^{2} d x d t+\frac{C_{N, s}}{2}\left\|u\left(x, t_{1}\right)\right\|_{X_{0}^{s}(\Omega)}^{2}-\lambda \int_{\Omega} \frac{u^{2}\left(x, t_{1}\right)}{|x|^{2 s}} d x \\
& -\frac{1}{1-\gamma} \int_{\Omega} u^{1-\gamma}\left(t_{1}\right) d x \\
\leq & \int_{t_{0}}^{t_{1}} \int_{\Omega} f \frac{\partial u}{\partial t} d x d t+\frac{C_{N, s}}{2}\left\|u\left(x, t_{0}\right)\right\|_{X_{0}^{s}(\Omega)}^{2}-\lambda \int_{\Omega} \frac{u^{2}\left(x, t_{0}\right)}{|x|^{2 s}} d x \\
& -\frac{1}{1-\gamma} \int_{\Omega} u^{1-\gamma}\left(t_{0}\right) d x .
\end{aligned}
$$

Noting that $u \in L^{\infty}\left([0, T] ; L^{p}(\Omega)\right)$ for $1<p<\infty$, we have

$$
\limsup _{t_{1} \rightarrow t_{0}^{+}}\left\|u\left(\cdot, t_{1}\right)\right\|_{X_{0}^{s}(\Omega)} \leq\left\|u\left(\cdot, t_{0}\right)\right\|_{X_{0}^{s}(\Omega)} \cdot
$$

Therefore (93), together with (90), gives $\lim _{t \rightarrow t_{0}^{+}}\|u(\cdot, t)\|_{X_{0}^{s}(\Omega)}=\left\|u\left(\cdot, t_{0}\right)\right\|_{X_{0}^{s}(\Omega)}$, which implies that $u$ is right continuous on $[0, T]$.

Now it suffices to prove the left continuity. Let $t_{1}>t_{0}$ and $0<r \leq t_{1}-t_{0}$. Define

$$
\left[\phi_{r}(u)\right](x, t):=\frac{u(x, t+r)-u(x, t)}{r} .
$$


Using $\phi_{r}(u)$ as the test function in (61), integrating over $\left(t_{0}, t_{1}\right) \times \mathbb{R}^{N}$, and using (77), (78), and (79), we get

$$
\begin{aligned}
\int_{t_{0}}^{t_{1}} & \int_{\Omega} \frac{\partial u}{\partial t} \phi_{r}(u) d x d t+\frac{C_{N, s}}{2 r} \int_{t_{0}}^{t_{1}} \int_{\mathbb{R}^{N}}\left(\left|(-\Delta)^{\frac{s}{2}} u(x, t+r)\right|^{2}-\left|(-\Delta)^{\frac{s}{2}} u(x, t)\right|^{2}\right) d x d t \\
& -\frac{\lambda}{r} \int_{t_{0}}^{t_{1}} \int_{\Omega} \frac{u^{2}(x, t+r)-u^{2}(x, t)}{|x|^{2 s}} d x d t \\
& -\frac{1}{r(1-\gamma)} \int_{t_{0}}^{t_{1}} \int_{\Omega}\left(u^{1-\gamma}(x, t+r)-u^{1-\gamma}(x, t)\right) d x d t \\
\geq & \int_{t_{0}}^{t_{1}} \int_{\Omega} f \phi_{r}(u) d x d t .
\end{aligned}
$$

Then an easy calculation gives

$$
\begin{aligned}
\int_{t_{0}}^{t_{1}} & \int_{\Omega} \frac{\partial u}{\partial t} \phi_{r}(u) d x d t+\frac{C_{N, s}}{2 r}\left(\int_{t_{1}}^{t_{1}+r} \int_{\mathbb{R}^{N}}\left|(-\Delta)^{\frac{s}{2}} u(x, t)\right|^{2} d x d t\right. \\
& \left.-\int_{t_{0}}^{t_{0}+r} \int_{\mathbb{R}^{N}}\left|(-\Delta)^{\frac{s}{2}} u(x, t)\right|^{2} d x d t\right) \\
& -\frac{\lambda}{r}\left(\int_{t_{1}}^{t_{1}+r} \int_{\Omega} \frac{u^{2}(x, t)}{|x|^{2 s}} d x d t-\int_{t_{0}}^{t_{0}+r} \int_{\Omega} \frac{u^{2}(x, t)}{|x|^{2 s}} d x d t\right) \\
& -\frac{1}{r(1-\gamma)}\left(\int_{t_{1}}^{t_{1}+r} \int_{\Omega} u^{1-\gamma}(x, t) d x d t-\int_{t_{0}}^{t_{0}+r} \int_{\Omega} u^{1-\gamma}(x, t) d x d t\right) \\
\geq & \int_{t_{0}}^{t_{1}} \int_{\Omega} f \phi_{r}(u) d x d t .
\end{aligned}
$$

Since $u(t) \in X_{0}^{S}(\Omega)$ is right continuous on $[0, T]$, using the dominated convergence theorem, as $r \rightarrow 0^{+}$, we get:

$$
\begin{aligned}
& \frac{1}{r} \int_{t_{1}}^{t_{1}+r} \int_{\mathbb{R}^{N}}\left|(-\Delta)^{\frac{s}{2}} u(x, t)\right|^{2} d x d t \rightarrow \int_{\mathbb{R}^{N}}\left|(-\Delta)^{\frac{s}{2}} u\left(x, t_{1}\right)\right|^{2} d x, \\
& \frac{1}{r} \int_{t_{0}}^{t_{0}+r} \int_{\mathbb{R}^{N}}\left|(-\Delta)^{\frac{s}{2}} u(x, t)\right|^{2} d x d t \rightarrow \int_{\mathbb{R}^{N}}\left|(-\Delta)^{\frac{s}{2}} u\left(x, t_{0}\right)\right|^{2} d x, \\
& \frac{1}{r} \int_{t_{1}}^{t_{1}+r} \int_{\Omega} \frac{u^{2}(x, t)}{|x|^{2 s}} d x d t \rightarrow \int_{\Omega} \frac{u^{2}\left(x, t_{1}\right)}{|x|^{2 s}} d x, \\
& \frac{1}{r} \int_{t_{0}}^{t_{0}+r} \int_{\Omega} \frac{u^{2}(x, t)}{|x|^{2 s}} d x d t \rightarrow \int_{\Omega} \frac{u^{2}\left(x, t_{0}\right)}{|x|^{2 s}} d x, \\
& \frac{1}{r} \int_{t_{1}}^{t_{1}+r} \int_{\Omega} u^{1-\gamma}(x, t) d x d t \rightarrow \int_{\Omega} u^{1-\gamma}\left(x, t_{1}\right) d x, \\
& \frac{1}{r} \int_{t_{0}}^{t_{0}+r} \int_{\Omega} u^{1-\gamma}(x, t) d x d t \rightarrow \int_{\Omega} u^{1-\gamma}\left(x, t_{0}\right) d x .
\end{aligned}
$$


Putting the results together in (94), as $r \rightarrow 0^{+}$, we obtain

$$
\begin{aligned}
\int_{t_{0}}^{t_{1}} & \int_{\Omega}\left(\frac{\partial u}{\partial t}\right)^{2} d x d t+\frac{C_{N, s}}{2}\left\|u\left(x, t_{1}\right)\right\|_{X_{0}^{s}(\Omega)}^{2}-\lambda \int_{\Omega} \frac{u^{2}\left(x, t_{1}\right)}{|x|^{2 s}} d x \\
& -\frac{1}{1-\gamma} \int_{\Omega} u^{1-\gamma}\left(t_{1}\right) d x \\
\geq & \int_{t_{0}}^{t_{1}} \int_{\Omega} f \frac{\partial u}{\partial t} d x d t+\frac{C_{N, s}}{2}\left\|u\left(x, t_{0}\right)\right\|_{X_{0}^{s}(\Omega)}^{2}-\lambda \int_{\Omega} \frac{u^{2}\left(x, t_{0}\right)}{|x|^{2 s}} d x \\
& -\frac{1}{1-\gamma} \int_{\Omega} u^{1-\gamma}\left(t_{0}\right) d x .
\end{aligned}
$$

Therefore (95) and (92) give the equality. Since the maps $t \mapsto \int_{\Omega} u^{1-\gamma}(x, t) d t$ and $t \mapsto$ $\int_{\Omega} \frac{u^{2}(x, t)}{|x|^{2 s}} d x$ are continuous, we have $u \in C\left([0, T] ; X_{0}^{s}(\Omega)\right)$. Moreover, we obtain (66) by taking $t_{1}=t$ and $t_{0}=0$.

Finally, we want to show that the solution obtained belongs to $C([0, T] ; W(\Omega))$ if the initial function $u_{0} \in \overline{\mathcal{D}(L)}^{L^{\infty}\left(\Omega,|x|^{\beta} d x\right)}$. We will use the $m$-accretive operator theory. Let $u_{0} \in$ $\overline{\mathcal{D}}(L)^{L^{\infty}\left(\Omega,|x|^{\beta} d x\right)}, \theta>0, f_{1}, f_{2} \in L^{\infty}\left(\Omega,|x|^{\beta} d x\right)$, and $0<\lambda<\lambda_{*}$. Also, let $u, v \in X_{0}^{s}(\Omega) \cap \mathcal{U}_{\gamma}^{\text {Sing }} \cap$ $W(\Omega)$ be the unique solutions to

$$
\begin{array}{ll}
u+\theta L(u)=f_{1} & \text { in } \Omega, \\
v+\theta L(v)=f_{2} & \text { in } \Omega .
\end{array}
$$

Note that the existence and uniqueness is guaranteed by Theorem 5.3. Subtracting the weak formulations of these two equations and using $w:=\left(|x|^{\beta}(u-v)-\left\|f_{1}-f_{2}\right\|_{L^{\infty}\left(\Omega,|x|^{\beta} d x\right)}\right)^{+}$ as a test function, we obtain

$$
\int_{\Omega} w^{2}|x|^{\beta} d x+\theta \int_{\Omega}(L(u)-L(v)) w d x \leq 0 .
$$

Since we can easily check that $\int_{\Omega}(L(u)-L(v)) w d x \geq 0$, we have $w \equiv 0$ a.e. in $\Omega$ or, equivalently, $|x|^{\beta}(u-v) \leq\left\|f_{1}-f_{2}\right\|_{L^{\infty}\left(\Omega,|x|^{\beta} d x\right)}$. Reversing the roles of $u$ and $v$ gives

$$
\|u-v\|_{L^{\infty}\left(\Omega,|x|^{\beta} d x\right)} \leq\left\|f_{1}-f_{2}\right\|_{L^{\infty}\left(\Omega,|x|^{\beta} d x\right)}
$$

This proves that $L$ is $m$-accretive in $W(\Omega)$. Now the rest of the proof van be obtained by invoking [58, Theorem 4.2], as explained in [31, Proposition 0.1].

Proof of Theorem 5.5 We follow the proof of [30, Theorem 2.12].

Let $\underline{u}, \bar{u} \in \overline{\mathcal{D}}(L)^{L^{\infty}\left(\Omega,|x|^{\beta} d x\right)}$ be the sub- and supersolutions, respectively, to (1) with $\mu \equiv 1$ such that $\underline{u} \leq u_{0} \leq \bar{u}$, which is possible because of $u_{0} \in \overline{\mathcal{D}}(L)^{L^{\infty}\left(\Omega,|x|^{\beta} d x\right)}$. Let $u$ be a weak solution of (61), and let $v_{1}$ and $v_{2}$ be the unique solutions to (61) with initial conditions $\underline{u}$ and $\bar{u}$, respectively. Since $\lambda \in\left(0, \lambda_{*}\right)$ and $\underline{u}, \bar{u} \in \overline{\mathcal{D}}(L)^{L^{\infty}\left(\Omega,|x|^{\beta} d x\right)}$, Theorem 5.4 gives $v_{1}, v_{2} \in C([0, T] ; W(\Omega))$. Taking $\underline{u}_{0}=\underline{u}$ (respectively, $\bar{u}_{0}=\bar{u}$ ), we consider the sequence $\left\{\underline{u}_{k}\right\}$ (respectively, $\left\{\bar{u}_{k}\right\}$ ) which is nondecreasing (respectively, nonincreasing) as solutions 
to the iteration given by (68). Moreover, we consider the sequence $\left\{u_{k}\right\}$ obtained in iteration (68) and starting with the initial condition $u_{0}$. Then by the choice of $\eta_{t}$ we have

$$
\underline{u}_{k} \leq u_{k} \leq \bar{u}_{k}
$$

which implies

$$
v_{1}(t) \leq u(t) \leq v_{2}(t)
$$

Now consider the maps $t \mapsto v_{1}(x, t)$ and $t \mapsto v_{2}(x, t)$, which are nondecreasing and nonincreasing, respectively (by similar reasoning as that in [59, Lemma 10.6] or in the proof of [30, Theorem 2.10]). Also, let $v_{1}(t) \rightarrow \tilde{v}_{1}$ and $v_{2}(t) \rightarrow \tilde{v}_{2}$ as $t \rightarrow \infty$. Moreover, if $S(t)$ denotes the semigroup on $W(\Omega)$ generated by the given evolution equation $u_{t}+L(u)=f(x)$, then we clearly have

$$
\tilde{v}_{1}=\lim _{t^{\prime} \rightarrow \infty} S\left(t^{\prime}+t\right)(\underline{u})=S(t) \lim _{t^{\prime} \rightarrow \infty} S\left(t^{\prime}\right)(\underline{u})=S(t) \lim _{t^{\prime} \rightarrow \infty} v_{1}\left(t^{\prime}\right)=S(t) \tilde{v}_{1}
$$

Similarly, we get

$$
\tilde{v}_{2}=S(t) \tilde{v}_{2}
$$

Thus $\tilde{v}_{1}$ and $\tilde{v}_{2}$ are stationary solutions to (61), that is, solve (1) with $\mu \equiv 1$. On the other hand, by the uniqueness of solutions to the stationary problem, $\tilde{v}_{1}=\tilde{v}_{2}=\hat{u}$. Now applying the Dini theorem (see [60, Theorem 7.13]) gives

$$
\left\{\begin{array}{l}
v_{1}(t) \rightarrow \hat{u}, \\
v_{2}(t) \rightarrow \hat{u},
\end{array} \text { in } L^{\infty}\left(\Omega,|x|^{\beta} d x\right) \text { as } t \rightarrow \infty .\right.
$$

Finally, using (96), we conclude that $u(t) \rightarrow \hat{u}$ in $L^{\infty}\left(\Omega,|x|^{\beta} d x\right)$ as $t \rightarrow \infty$.

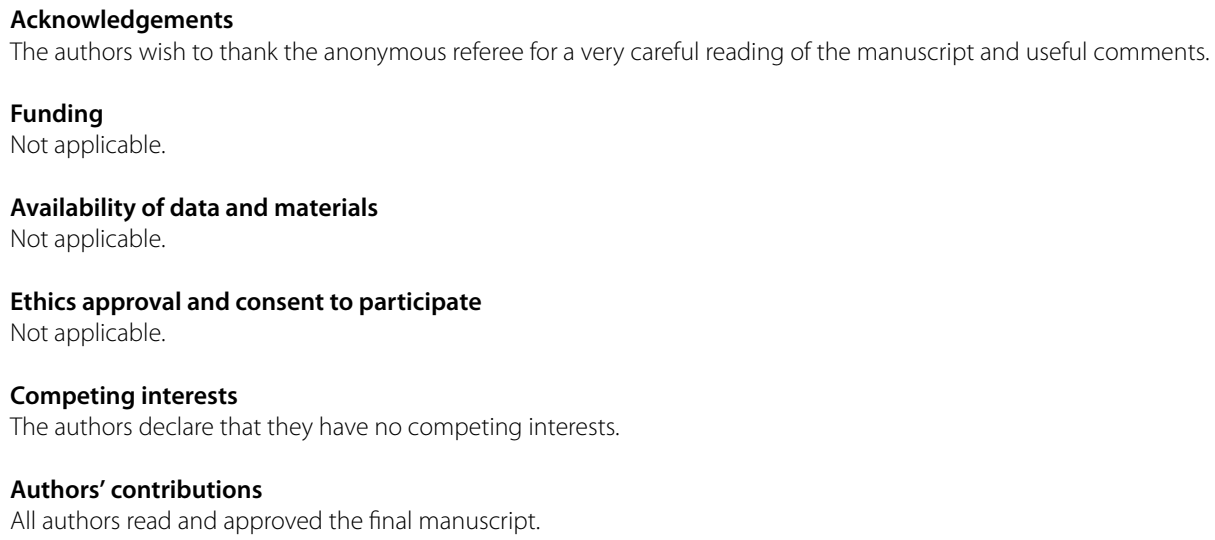




\section{Publisher's Note}

Springer Nature remains neutral with regard to jurisdictional claims in published maps and institutional affiliations.

\section{Received: 14 March 2021 Accepted: 21 July 2021 Published online: 31 July 2021}

\section{References}

1. Boumediene, A., Peral, I., Primo, A.: Influence of the Hardy potential in a semilinear heat equation. Proc. R. Soc. Edinb., Sect. A 139(5), 897-926 (2009)

2. Pérez-Llanos, M., Primo, A.: Semilinear biharmonic problems with a singular term. J. Differ. Equ. 257(9), 3200-3225 (2014)

3. Abatangelo, N., Valdinoci, E.: Getting acquainted with the fractional Laplacian. In: Contemporary Research in Elliptic PDEs and Related Topics. Springer INdAM Ser., vol. 33, pp. 1-105. Springer, Cham (2019)

4. Di Nezza, E., Palatucci, G., Valdinoci, E.: Hitchhiker's guide to the fractional Sobolev spaces. Bull. Sci. Math. 136(5), 521-573 (2012)

5. Silvestre, L.: Regularity of the obstacle problem for a fractional power of the Laplace operator. Commun. Pure Appl. Math. 60(1), 67-112 (2007)

6. Ghergu, M., Taliaferro, S.D.: Isolated Singularities in Partial Differential Inequalities. Encyclopedia of Mathematics and Its Applications, vol. 161. Cambridge University Press, Cambridge (2016)

7. Ghergu, M., Rădulescu, V.D.: Singular Elliptic Problems: Bifurcation and Asymptotic Analysis. Oxford Lecture Series in Mathematics and Its Applications, vol. 37. The Clarendon Press, Oxford (2008)

8. Gazzola, F., Grunau, H.-C., Mitidieri, E.: Hardy inequalities with optimal constants and remainder terms. Trans. Am. Math. Soc. 356(6), 2149-2168 (2004)

9. Mitidieri, E.: A simple approach to Hardy inequalities. Mat. Zametki 67(4), 563-572 (2000)

10. Alonso, I.P., de Diego, F.S.: Elliptic and Parabolic Equations Involving the Hardy-Leray Potential. Walter de Gruyter GmbH \& Co KG, Berlin (2021)

11. Biccari, U.: On the controllability of partial differential equations involving non-local terms and singular potentials. Dissertation, Universidad del País Vasco-Euskal Herriko Unibertsitatea (2016)

12. Baras, P., Goldstein, J.A.: The heat equation with a singular potential. Trans. Am. Math. Soc. 284(1), 121-139 (1984)

13. Baras, P., Goldstein, J.A.: Remarks on the inverse square potential in quantum mechanics. In: Differential Equations, Birmingham, Ala., 1983. North-Holland Math. Stud., vol. 92, pp. 31-35. North-Holland, Amsterdam (1984)

14. Yafaev, D.: Sharp constants in the Hardy-Rellich inequalities. J. Funct. Anal. 168(1), 121-144 (1999)

15. Boccardo, L., Orsina, L.: Semilinear elliptic equations with singular nonlinearities. Calc. Var. Partial Differ. Equ. 37(3-4), 363-380 (2010)

16. Orsina, L., Petitta, F.: A Lazer-McKenna type problem with measures. Differ. Integral Equ. 29(1-2), 19-36 (2016)

17. Barrios, B.N., De Bonis, I., Medina, M., Peral, I.: Semilinear problems for the fractional Laplacian with a singular nonlinearity. Open Math. 13(1), 390-407 (2015)

18. Canino, A., Montoro, L., Sciunzi, B., Squassina, M.: Nonlocal problems with singular nonlinearity. Bull. Sci. Math. 141(3), 223-250 (2017)

19. De Cave, L.M., Durastanti, R., Oliva, F.: Existence and uniqueness results for possibly singular nonlinear elliptic equations with measure data. NoDEA Nonlinear Differ. Equ. Appl. 25(3), 18-35 (2018)

20. Giacomoni, J., Mukherjee, T., Sreenadh, K.: Existence of three positive solutions for a nonlocal singular Dirichlet boundary problem. Adv. Nonlinear Stud. 19(2), 333-352 (2019)

21. Lazer, A.C., McKenna, P.J.: On a singular nonlinear elliptic boundary-value problem. Proc. Am. Math. Soc. 111(3), 721-730 (1991)

22. Molica Bisci, G., Repovš, D.: Existence and localization of solutions for nonlocal fractional equations. Asymptot. Anal. 90(3-4), 367-378 (2014)

23. Oliva, F., Petitta, F.: On singular elliptic equations with measure sources. ESAIM Control Optim. Calc. Var. 22(1), 289-308 (2016)

24. Oliva, F., Petitta, F.: Finite and infinite energy solutions of singular elliptic problems: existence and uniqueness. J. Differ. Equ. 264(1), 311-340 (2018)

25. Arora, R., Giacomoni, J., Warnault, G.: Regularity results for a class of nonlinear fractional Laplacian and singular problems. NoDEA Nonlinear Differ. Equ. Appl. 28(3), 30-35 (2021). https://doi.org/10.1007/s00030-021-00693-9

26. Marta, L., Oliva, F., Petitta, F., de León, S.S.: The Dirichlet problem for the 1-Laplacian with a general singular term and $L^{1}$-data. Nonlinearity 34(3), 1791-1816 (2021)

27. Nachman, A., Callegari, A.J.: A nonlinear singular boundary value problem in the theory of pseudoplastic fluids. SIAM J. Appl. Math. 38(2), 275-281 (1980). https://doi.org/10.1137/0138024

28. Mukherjee, T., Sreenadh, K.: Fractional elliptic equations with critical growth and singular nonlinearities. Electron. J. Differ. Equ. 2016, 54 (2016)

29. Boumediene, A., Medina, M., Peral, I., Primo, A.: The effect of the Hardy potential in some Calderón-Zygmund properties for the fractional Laplacian. J. Differ. Equ. 260(11), 8160-8206 (2016)

30. Giacomoni, J., Mukherjee, T., Sreenadh, K.: Existence and stabilization results for a singular parabolic equation involving the fractional Laplacian. Discrete Contin. Dyn. Syst., Ser. S 12(2), 311-337 (2019)

31. Badra, M., Bal, K., Giacomoni, J.: A singular parabolic equation: existence, stabilization. J. Differ. Equ. 252(9), 5042-5075 (2012)

32. Bougherara, B., Giacomoni, J.: Existence of mild solutions for a singular parabolic equation and stabilization. Adv. Nonlinear Anal. 4(2), 123-134 (2015)

33. Giacomoni, J., Goel, D., Sreenadh, K.: Singular doubly nonlocal elliptic problems with Choquard type critical growth nonlinearities (2020). arXiv:2002.02937

34. Giacomoni, J., Kumar, D., Sreenadh, K.: Sobolev and Hölder regularity results for some singular double phase problems (2020). arXiv:2004.06699

35. Servadei, R., Valdinoci, E.: Mountain pass solutions for non-local elliptic operators. J. Math. Anal. Appl. 389(2), 887-898 (2012) 
36. Molica Bisci, G., Repovš, D.: On doubly nonlocal fractional elliptic equations. Atti Accad. Naz. Lincei, Rend. Lincei, Mat. Appl. 26(2), 161-176 (2015)

37. Ros-Oton, X., Serra, J.: The Dirichlet problem for the fractional Laplacian: regularity up to the boundary. J. Math. Pures Appl. (9) 101(3), 275-302 (2014)

38. Servadei, R., Valdinoci, E.: Variational methods for non-local operators of elliptic type. Discrete Contin. Dyn. Syst. 33(5), 2105-2137 (2013)

39. Barrios, B.N., Medina, M.: Strong maximum principles for fractional elliptic and parabolic problems with mixed boundary conditions. Proc. R. Soc. Edinb., Sect. A 150(1), 475-495 (2020). https://doi.org/10.1017/prm.2018.77

40. Barrios, B., Medina, M., Peral, I.: Some remarks on the solvability of non-local elliptic problems with the Hardy potential. Commun. Contemp. Math. 16(4), 1350046 (2014)

41. Boccardo, L., Orsina, L., Peral, I.: A remark on existence and optimal summability of solutions of elliptic problems involving Hardy potential. Discrete Contin. Dyn. Syst. 16(3), 513-523 (2006)

42. Boumediene, A., Medina, M., Peral, I., Primo, A.: Optimal results for the fractional heat equation involving the Hardy potential. Nonlinear Anal. 140, 166-207 (2016)

43. Papageorgiou, N.S., Rădulescu, V.D., Repovš, D.D.: Nonlinear Analysis-Theory and Methods. Springer Monographs in Mathematics. Springer, Cham (2019)

44. Leonori, T., Peral, I., Primo, A., Soria, F.: Basic estimates for solutions of a class of nonlocal elliptic and parabolic equations. Discrete Contin. Dyn. Syst. 35(12), 6031-6068 (2015)

45. Boumediene, A., Peral, I., Primo, A.: A remark on the fractional Hardy inequality with a remainder term. C. R. Math. Acad. Sci. Paris 352(4), 299-303 (2014)

46. Huyuan, C., Felmer, P., Véron, L.: Elliptic equations involving general subcritical source nonlinearity and measures (2014). arXiv:1409.3067

47. Marcus, M., Véron, L.: Nonlinear Second Order Elliptic Equations Involving Measures. De Gruyter Series in Nonlinear Analysis and Applications, vol. 21. de Gruyter, Berlin (2014)

48. Stein, E.M.: Singular Integrals and Differentiability Properties of Functions. Princeton Mathematical Series, vol. 30. Princeton University Press, Princeton (1970)

49. Adimurthi, G.J., Santra, S.: Positive solutions to a fractional equation with singular nonlinearity. J. Differ. Equ. 265(4), 1191-1226 (2018)

50. Brasco, L., Cinti, E.: On fractional Hardy inequalities in convex sets. Discrete Contin. Dyn. Syst. 38(8), 4019-4040 (2018)

51. Boumediene, A., Biroud, K., Primo, A.: Nonlinear fractional elliptic problem with singular term at the boundary. Complex Var. Elliptic Equ. 64(6), 909-932 (2019)

52. Dyda, B.: A fractional order Hardy inequality. III. J. Math. 48(2), 575-588 (2004)

53. Filippas, S., Moschini, L., Tertikas, A.: Sharp trace Hardy-Sobolev-Maz'ya inequalities and the fractional Laplacian. Arch. Ration. Mech. Anal. 208(1), 109-161 (2013)

54. Bénilan, P., Boccardo, L., Gallouët, T., Gariepy, R., Pierre, M., Vázquez, J.L.: An L' '-theory of existence and uniqueness of solutions of nonlinear elliptic equations. Ann. Sc. Norm. Super. Pisa, Cl. Sci. (4) 22(2), 241-273 (1995)

55. Boccardo, L., Gallouët, T., Orsina, L.: Existence and uniqueness of entropy solutions for nonlinear elliptic equations with measure data. Ann. Inst. Henri Poincaré, Anal. Non Linéaire 13(5), 539-551 (1996)

56. Simon, J.: Compact sets in the space $L^{P}(0, T ; B)$. Ann. Mat. Pura Appl. (4) 146, 65-96 (1987)

57. Badiale, M., Serra, E.: Semilinear Elliptic Equations for Beginners: Existence Results via the Variational Approach. Universitext. Springer, London (2011)

58. Barbu, V:: Nonlinear Differential Equations of Monotone Types in Banach Spaces. Springer Monographs in Mathematics. Springer, New York (2010)

59. Smoller, J.: Shock Waves and Reaction-Diffusion Equations, 2nd edn. Grundlehren der Mathematischen Wissenschaften [Fundamental Principles of Mathematical Sciences], vol. 258. Springer, New York (1994). https://doi.org/10.1007/978-1-4612-0873-0

60. Rudin, W.: Principles of Mathematical Analysis, 3rd edn. International Series in Pure and Applied Mathematics, McGraw-Hill, New York (1976)

\section{Submit your manuscript to a SpringerOpen ${ }^{\circ}$ journal and benefit from:}

- Convenient online submission

- Rigorous peer review

- Open access: articles freely available online

- High visibility within the field

- Retaining the copyright to your article

Submit your next manuscript at $\gg$ springeropen.com 